\title{
Non-Abelian Magnetic Monopoles ${ }^{\star}$
}

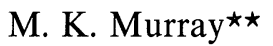 \\ Mathematical Sciences Research Institute, 2223 Fulton Street, Room 603, Berkeley, CA 94720, \\ USA
}

\begin{abstract}
It is shown that a general, irreducible, $\mathrm{SU}(n), \mathrm{Sp}(n), \mathrm{SO}(2 n)$ monopole with maximal symmetry breaking is determined by its spectral data. For SU(n) with minimal symmetry breaking the spectral data is defined and also shown to determine the monopole.
\end{abstract}

\section{Introduction}

In a previous paper [12] the definition of the spectral curve of a monopole, given in [9] by Hitchin for SU(2), was extended to any compact, connected, simple Lie group $K$. In this paper the details of the results announced in [12] are presented. It is shown that there are $r=\operatorname{rank} K$ spectral curves $S_{1}, \ldots, S_{r}$ for a $K$ monopole. The spectral curves are labelled by the simple roots $\left\{\alpha_{1}, \ldots, \alpha_{r}\right\}$, and when $\alpha_{i}$ and $\alpha_{j}$ are joined on the Dynkin diagram the intersection $S_{i} \cap S_{j}$ has a splitting as $S_{i} \cap S_{j}=S_{i j}$ $\cup S_{j i}$. The curves and this splitting constitute the spectral data of the monopole. The main result of this paper is that for $\mathrm{SU}(n), \mathrm{SO}(2 n)$ and $\mathrm{Sp}(n)$ an irreducible, general monopole is determined by its spectral data.

In Sect. 1 the basic material on monopoles and the definition of the magnetic charges $\left\{m_{1}, \ldots, m_{r}\right\}$ of a monopole, with maximal symmetry breaking at infinity, are reviewed. The definition of the twistor space $\mathscr{T}$ is also recalled and used in Sect. 2 to generalize the twistor correspondence of Hitchin and Ward. The general twistor correspondence associates to any $K$ monopole with reduction at infinity to a maximal torus $T$, a holomorphical principal bundle $Q$, on $\mathscr{T}$, with structure group $G$, the complexification of $K$, and two reductions $R^{+}, R^{-} \subset Q$ to Borel subgroups of $G$.

* Research supported in part by NSF Grant 8120790

$\star \star$ Present address: Department of Mathematics, Research School of Physical Sciences, Australian National University, GPO Box 4, Canberra, Australia 2601 
The spectral data is defined in Sect. 3 by combining two different approaches. The first concentrates on the representation theory of $K$ and realizes the spectral curves $S_{1}, \ldots, S_{r}$ as sections of line bundles of degree $2 m_{i}$. This incidentally shows that the magnetic charges must be non-negative. The second approach uses the Bruhat cell decomposition of $G / B$ and determines the decomposition $S_{i} \cap S_{j}=S_{i j}$ $\cup S_{j i}$.

In Sect. 4 the standard representations of $\mathrm{SU}(n), \mathrm{SO}(2 n)$ and $\mathrm{Sp}(n)$ are used to define a vector bundle associated to $Q$ with a flag. The method used by Hitchin in [9] to reconstruct the $\mathrm{SU}(2)$ monopole can be applied to each step of the flag to reconstruct the bundle.

The last section considers non-maximal symmetry breaking. In particular for $\mathrm{SU}(n)$ with minimal symmetry breaking it is shown that the spectral data, a curve $S$ and a section of a vector bundle over $S$, determine the monopole.

\section{Review}

A magnetic monopole is a solution of the Bogomolny equations that satisfies the Bogomonly-Prasad-Sommerfield (BPS) boundary conditions.

We start with a principal $K$ bundle $P \rightarrow \mathbb{R}^{3}$, where here and throughout $K$ is a compact, connected, simple Lie group. A monopole configuration is a pair $(A, \Phi)$ where $A$ is a connection for the bundle $P$ and $\Phi$ is a section of the adjoint bundle, called a Higgs field. These satisfy the Bogomolny equations if

$$
{ }^{*} F_{A}=d_{A} \Phi,
$$

where $F_{A}$ is the curvature of $A, d_{A}$ the exterior covariant derivative and * the duality operator on $\mathbb{R}^{3}$.

The BPS boundary conditions essentially require that at infinity the bundle $P$ reduces to a sub-bundle determined by the Higgs field. This reduction is obtained by requiring that for any choice of origin and radial gauge around it, that is a section $s$ of $P$ such that $\left\langle s^{*} A, \frac{\partial}{\partial r}\right\rangle=0$ where $(r, \theta, \phi)$ are polar coordinates, the limiting map $\Phi^{\infty}: S_{\infty}^{2} \rightarrow L K$, defined by $\Phi^{\infty}(\theta, \phi)=\lim _{r \rightarrow \infty} s^{*} \Phi(r, \theta, \phi)$ exists and its image is contained in an orbit of the adjoint action of $K$ on $L K$. Such orbits have the form $K / C\left(T_{0}\right)$, where $C\left(T_{0}\right)$ is the centralizer of a torus $T_{0}$ in $K$. Generically $T_{0}$ $=T$ a maximal torus so the orbit is just $K / T$. The subgroup $C\left(T_{0}\right)$ is the isotropy subgroup of $\Phi^{\infty}$ evaluated at some point on $S_{\infty}^{2}$ the two sphere at infinity. The Higgs field at infinity therefore determines a section of $P^{\infty}\left(K / C\left(T_{0}\right)\right)=P^{\infty}$ $\times_{K} K / C\left(T_{0}\right)$, where $P^{\infty}$ is the bundle at infinity, and by a standard result [11] this means that $P^{\infty}$ has a reduction to a $C\left(T_{0}\right)$ sub-bundle. This sub-bundle is isomorphic to the pullback by $\Phi^{\infty}$ of the standard $C\left(T_{0}\right)$ bundle $K \rightarrow K / C\left(T_{0}\right)$.

In a radial gauge $s$ the connection $s^{*} A$ takes the form $s^{*} A=A_{\theta} d \theta+A_{\phi} d \phi$. If the limits of $A_{\theta}, A_{\phi}$ exist we say the connection has a limit on the bundle at infinity and likewise for the curvature.

Now the Bogomolny-Prasad-Sommerfield (BPS) boundary conditions are:

BPS 1. The Higgs field at infinity, $\Phi^{\infty}$, exists and is at least $C^{1}$ and the connection at infinity, $A^{\infty}$, exists and is at least $C^{2}$ with curvature given by $F^{\infty}$. 
BPS 2. For any line $\gamma$ in $\mathbb{R}^{3}$ if we choose orthogonal coordinates $t$, along $\gamma$, and $e_{1}, e_{2}$ and a gauge $s$ in a neighborhood of $\gamma$ such that $\left\langle s^{*} A, \frac{\partial}{\partial t}\right\rangle=0$, then the limits of $A_{1}$ and $A_{2}$ exist and are at least $C^{2}$ where $s^{*} A=A_{1} d e_{1}+A_{2} d e_{2}$.

BPS 3. For some $x \in \mathbb{R}^{3}$ and choice of radial gauge $s$ we have a uniform expansion of $S^{*} \Phi$ outside of some compact set as

where $\psi: S_{\infty}^{2} \rightarrow L K$.

$$
s^{*} \Phi=\Phi^{\infty}+\frac{1}{r} \psi+O\left(\frac{1}{r^{2}}\right),
$$

BPS $4 . \Phi^{\infty}$ lies in an orbit of the action of $K$ on $L K$.

BPS 5. $\left|d_{A} \Phi\right|=O\left(\frac{1}{r^{2}}\right)$.

BPS 6. $\frac{\partial|\Phi|}{\partial \Omega}=O\left(\frac{1}{r^{2}}\right)$, where in polar coordinates

$$
\left(\frac{\partial|\Phi|}{\partial \Omega}\right)^{2}=\left(\frac{\partial|\Phi|}{\partial \theta}\right)^{2}+\sin ^{2} \theta\left(\frac{\partial|\Phi|}{\partial \phi}\right)^{2} .
$$

These conditions are independent of the choice of origin and radial gauge. This is obvious for all except BPS 3. But if we look at the Bogomolny equations it is easy to deduce that $\psi=-{ }^{*} F^{\infty}$, where $*$ is the duality operator on $S_{\infty}^{2}$. So BPS 3 says that $\left|s^{*} \Phi-\Phi^{\infty}+{ }^{*} F^{\infty} \frac{1}{r}\right|$ falls off as $O\left(\frac{1}{r^{2}}\right)$, which is independent of the choice of origin.

The first two of these conditions are rather technical but are necessary to prove Theorem 21. It seems plausible that by using the Bogomolny equations they could be deduced from BPS 3, 4, 5, 6. Taubes (unpublished) has shown that BPS 5, 6 follows from BPS 3, [10].

Until we get to Sect. 5 we will assume that the isotropy subgroup of $\Phi^{\infty}$ is a maximal torus, that is, the magnetic monopole has maximal symmetry breaking at infinity.

The Higgs field at infinity defines a homotopy class $\left[\Phi^{\infty}\right] \in \Pi_{2}(K / T)$ which is independent of the choice of radial gauge because $K$ is connected. As the Bogomolny equations are defined in the adjoint representation we can assume, for simplicity, that $K$ is simply connected. Then from the homotopy exact sequence of $T \rightarrow K \rightarrow K / T$ we have

where $r$ is the rank of $K$.

$$
\Pi_{2}(K / T) \simeq \Pi_{1}(T) \simeq \mathbb{Z}^{r},
$$

In Sect. 3 we will discuss the topology of $K / T$ in some detail but it is useful to review the two-dimensional topology here. The homotopy group $\Pi_{2}(K / T)$ has a natural basis $\left\{c_{1}, \ldots, c_{r}\right\}$ labelled by the simple roots $\left\{\alpha_{1}, \ldots, \alpha_{r}\right\}$ of $K$ with respect to some fundamental Weyl chamber. If $\Lambda \subset L T^{*}$ is the lattice of weights, then each $\lambda \in \Lambda$ defines a representation $\lambda: T \rightarrow \mathrm{U}(1)$ and therefore a line bundle $\mathscr{L}^{\lambda}$ associated to the principal bundle $K(K / T, T)$. Taking the Chern class of $\mathscr{L}^{-\lambda}$ defines an isomorphism [3]

$$
\Lambda \otimes_{\mathbb{Z}} \mathbb{Q} \rightarrow H^{2}(K / T, \mathbb{Q}), \quad \lambda \mapsto c\left(\mathscr{L}^{-\lambda}\right) .
$$


The natural pairing of $[f] \in \Pi_{2}(K / T)$ and $c\left(\mathscr{L}^{-\lambda}\right) \in H^{2}(K / T, \mathbb{Q})$ is then

$$
\left\langle[f], c\left(\mathscr{L}^{-\lambda}\right)\right\rangle=c\left(f^{*} \mathscr{L}^{-\lambda}\right) \in H^{2}\left(S^{2}\right) \simeq \mathbb{Q} .
$$

In the basis $\left\{c_{1}, \ldots, c_{r}\right\}$ this is also given by [3]

$$
\left\langle c_{i}, c\left(\mathscr{L}^{-\lambda}\right)\right\rangle=\frac{2\left\langle\lambda, \alpha_{i}\right\rangle}{\left\langle\alpha_{i}, \alpha_{i}\right\rangle},
$$

where $\langle$,$\rangle is the Killing form.$

Then the topological charges $m_{1}, \ldots, m_{r}$ are defined by

$$
\left[\Phi^{\infty}\right]=\sum_{i=1}^{r} m_{i} c_{i} .
$$

Here we choose some point $N \in S_{\infty}^{2}$, fix the torus $T$ to be the isotropy subgroup of $\Phi^{\infty}(N)$ and let the fundamental Weyl chamber be the one containing $\Phi^{\infty}(N)$. If $\left\{\lambda_{1}, \ldots, \lambda_{r}\right\}$ are the fundamental weights defined by

$$
\frac{2\left\langle\lambda_{i}, \alpha_{j}\right\rangle}{\left\langle\alpha_{j}, \alpha_{j}\right\rangle}=\delta_{i j},
$$

then

$$
m_{i}=\left\langle\left[\Phi^{\infty}\right], c\left(\mathscr{L}^{-\lambda_{i}}\right)\right\rangle=c\left(\Phi^{\infty *}\left(\mathscr{L}^{-\lambda_{i}}\right)\right) .
$$

Using the fact that $\left(\Phi^{\infty}\right)^{*}(K)$ is the reduced bundle at infinity and that the connection reduces we have

$$
c\left(\Phi^{\infty *} \mathscr{L}^{-\lambda_{i}}\right)=\frac{-i}{2 \pi} \int_{S^{2}} i \lambda_{i}\left({ }^{*} F^{\infty}\right) \operatorname{vol}\left(S^{2}\right)=2 \lambda_{i}\left({ }^{*} F^{\infty}\right),
$$

and therefore $m_{i}=2 \lambda_{i}\left({ }^{*} F^{\infty}\right)$.

We see from these results that the topological properties of $\left[\Phi^{\infty}\right] \in \Pi_{2}(K / T)$ can be obtained from ${ }^{*} F^{\infty}$ evaluated along any direction in $\mathbb{R}^{3}$. We will often abuse notation and write $\Phi^{\infty}$ or ${ }^{*} F^{\infty}$ when we mean $\Phi^{\infty}(N),{ }^{*} F^{\infty}(N)$ for some $N \in S_{\infty}^{2}$.

Using the Lagrangian from which the equations are derived [4] it is not hard to show that the topological charges are constrained by the requirement that

$$
\left\langle{ }^{*} F^{\infty}, \Phi^{\infty}\right\rangle \geqq 0 .
$$

We will show in Sect. 3 that they must, in fact, be all nonnegative.

To define spectral curves for magnetic monopoles for arbitrary groups we need to use the twistor method of Hitchin and Ward [9].

Their twistor correspondence is a correspondence between SU(2) monopoles and certain holomorphic bundles over a complex manifold $\mathscr{T}$. The twistor space $\mathscr{T}$ is the space of all oriented lines in $\mathbb{R}^{3}$. If $\gamma$ is such a line there is a unique unit vector $\hat{\mathbf{u}}$ in the direction of $\gamma$ and a unique shortest vector $\mathbf{v}$ from the origin $\mathbf{0} \in \mathbb{R}^{3}$ to $\gamma$. So

$$
\mathscr{T}=\{(\hat{\mathbf{u}}, \mathbf{v}) \mid \hat{\mathbf{u}} \cdot \hat{\mathbf{u}}=1, \mathbf{v} \cdot \hat{\mathbf{u}}=0\}=T S^{2} \cong T \mathbb{P}_{1},
$$

and therefore $\mathscr{T}$ is a two dimensional complex manifold. 
This complex structure can also be described intrinsically. The space $\mathscr{T}$ is part of the double fibration (cf. [7])

$$
\begin{array}{ll}
\multicolumn{2}{c}{S^{2} \times \mathbb{R}^{3}} \\
p \swarrow \quad \searrow \pi \\
\mathbb{R}^{3} \quad \mathscr{T}
\end{array}
$$

where $p$ projects onto $\mathbb{R}^{3}$ and $\pi(\hat{\mathbf{u}}, \mathbf{v})=\{\hat{\mathbf{u}}+t \mathbf{v} \mid t \in \mathbb{R}\}$. The fibres of $\pi$ are the orbits of the action of $\mathbb{R}$ on $S^{2} \times \mathbb{R}^{3}$ given by $(\hat{\mathbf{u}}, \mathbf{v}) \mapsto(\hat{\mathbf{u}}, t \hat{\mathbf{u}}+\mathbf{v})$, for $t \in \mathbb{R}$, and induce a vector field $X$, the "geodesic flow", on $S^{2} \times \mathbb{R}^{3}$.

With the natural metric on $S^{2} \times \mathbb{R}^{3}$ the tangent bundle splits into a onedimensional bundle parallel to the geodesic flow and an orthogonal bundle which has a natural complex structure obtained from the complex structure on $S^{2}$ and cross-product with the geodesic flow $X$. This complex structure is invariant under the geodesic flow and projects onto the complex structure on $\mathscr{T}$ [9].

The twistor space has an antiholomorphic involution $\tau: \mathscr{T} \rightarrow \mathscr{T}$ given by reversing the orientation on each line. A subset $S$ of $\mathscr{T}$ is called real if $\tau(S)=S$. There are no real points on $\mathscr{T}$ but there are real "lines." These are determined by picking a point $x$ in $\mathbb{R}^{3}$ and letting

$$
p_{x}=\{\gamma \in \mathscr{T} \mid \gamma \text { goes through } x\},
$$

which defines a real holomorphic curve on $\mathscr{T}$ [9]. In terms of the double fibration, if $x \in \mathbb{R}^{3}, p_{x}=\pi\left(p^{-1}(x)\right)$ and if $\gamma \in \mathscr{T}$, the corresponding line is $\gamma=p\left(\pi^{-1}(\gamma)\right)$. So we have the basic correspondence

$$
\begin{gathered}
\text { \{oriented lines in } \left.\mathbb{R}^{3}\right\} \leftrightarrow\{\text { points in } \mathscr{T}\}, \\
\text { \{points in } \left.\mathbb{R}^{3}\right\} \leftrightarrow\{\text { real lines in } \mathscr{T}\} .
\end{gathered}
$$

In holomorphic terms $\mathscr{T} \simeq T \mathbb{P}_{1}$ and we can define the bundle $O(k)$ on $\mathscr{T}$ by pulling back $O(k)$ on $\mathbb{P}_{1}$. Then the real lines $p_{x}$ are divisors of real sections of $O(2)$ [9].

The choice of origin in $\mathbb{R}^{3}$ is arbitrary and we can regard $\mathscr{T}$ as an affine bundle over $\mathbb{P}_{1}$. A choice of zero section corresponds to a choice of origin and vice versa.

\section{Twistor Correspondence}

The twistor correspondence of Hitchin and Ward [9] relates monopoles for an $\mathrm{SU}(2)$ vector bundle on $\mathbb{R}^{3}$ and holomorphic vector bundles on twistor space $\mathscr{T}$. It will be useful for our purposes to derive the equivalent correspondence for principal bundles.

We recall first that any compact, connected Lie group $K$ has a complexification $G$. This is a complex Lie group containing $K$ as a subgroup and whose Lie algebra $L G$ is the complexification of the Lie algebra of $K$. The basic example is $K=\mathrm{SU}(n)$ and $G=\operatorname{SL}(n, \mathbb{C})$. In this case we note that the map $X \mapsto\left(X^{*}\right)^{-1}$ is an antiholomorphic automorphism of $\mathrm{SL}(n, \mathbb{C})$ whose fixed point set is $\mathrm{SU}(n)$. In general such a real structure always exists and we will denote it by $g \mapsto \bar{g}$.

To define a holomorphic structure on a complex vector bundle $E$ over a complex manifold $\mathscr{T}$ it is necessary and sufficient to define an integrable 
$\bar{\partial}$-operator. A $\bar{\partial}$-operator is a $\mathbb{C}$ linear map

$$
\bar{\partial}: \Gamma(E) \rightarrow \Gamma(E) \otimes T^{0,1}(\mathscr{T})
$$

satisfying $\bar{\partial}(f s)=\bar{\partial} f \otimes s+f \otimes \bar{\partial} s$ for all $f \in C^{\infty}(\mathscr{T})$ and $s \in \Gamma(E)$. Such an operator is said to be integrable if $\bar{\partial}^{2}=0$. It is then a corollary of the Newlander-Nirenberg theorem (see [2]) that this determines a holomorphic structure on $E$ for which it is the natural $\bar{\partial}$-operator.

In the case of a principal bundle with total space $Q$, base space $\mathscr{T}$ and structure group $G$ a complex Lie group, an analogous result holds. Such a bundle is holomorphic if $Q$ is a complex manifold, the $G$ action is holomorphic and $Q \rightarrow \mathscr{T}$ admits holomorphic local sections. Alternatively, to determine a holomorphic structure on the bundle it suffices to choose a cover $\left\{U_{\alpha}\right\}$ of $\mathscr{T}$ and local sections $s_{\alpha}: U_{\alpha} \rightarrow Q$ such that the transition functions $g_{\alpha \beta}: U_{\alpha} \cap U_{\beta} \rightarrow G$ are holomorphic.

By analogy with a connection a $\bar{\partial}$-connection can be defined as a distribution of subspaces of the complexified tangent space of $Q$ or as a certain 1-form on $Q$. For our purposes it will be most useful to have the following local definition.

Definition (15). A $\bar{\partial}$-connection is a collection of 1-forms $\left\{A_{\alpha}\right\}$ with $A_{\alpha} \in \Gamma\left(U_{\alpha}\right.$, $\left.L G \otimes T^{0,1}\right)$ satisfying

$$
A_{\beta}=g_{\alpha \beta} A g_{\alpha \beta}^{-1}+g_{\alpha \beta} \bar{\partial} g_{\alpha \beta}^{-1} \text {. }
$$

If the $\left\{g_{\alpha \beta}\right\}$ are holomorphic there is a natural $\bar{\partial}$-connection obtained by taking all $A_{\alpha}$ to be zero. Given a $\bar{\partial}$-connection we can define a global $(0,2)$ form $F \in \Gamma\left(\mathscr{T}, \operatorname{ad} Q \otimes \Lambda^{0,2} T^{*} \mathscr{T}\right)$ with local coordinates

$$
F_{\alpha}=\bar{\partial} A_{\alpha}+A_{\alpha} \wedge A_{\alpha},
$$

and the $\bar{\partial}$-connection is said to be integrable if $F \equiv 0$. In such a case it again follows from the Newlander-Nirenberg theorem that we can choose a holomorphic structure on $Q$ so that this $\bar{\partial}$-connection is the natural one (see [2]).

Now we start with a principal $K$ bundle $P \rightarrow \mathbb{R}^{3}$ with a connection $A$ and Higgs field $\Phi$ and define a principal $G$ bundle $Q \rightarrow \mathscr{T}$ with fibre over $\gamma \in \mathscr{T}$ given by

$$
Q(\gamma)=\left\{s \in \Gamma\left(\gamma, P^{c}\right) \mid\left\langle s^{*} A, \gamma_{*}\right\rangle=i s^{*} \Phi\right\} .
$$

Here $P^{c}=P \times{ }_{K} G$ is the complexification of $P$ and $\gamma_{*}$ the unit tangent vector field to the geodesic $\gamma$ in the direction of orientation.

We can now generalize Hitchin's theorem (4.2) to obtain:

Theorem (18). If $(A, \Phi)$ is a solution of the Bogomolny equations $Q$ has a holomorphic structure and an antiholomorphic map $\sigma: Q \rightarrow Q$ covering $\tau: \mathscr{T} \rightarrow \mathscr{T}$, satisfying

(1) $Q$ is holomorphically trivial on real sections,

(2) if $q \in Q, g \in G$ then $\sigma(q g)=\sigma(q) \bar{g}$,

(3) $\sigma^{2}=1$.

Conversely such a bundle defines a solution of the Bogomolny equations.

Proof. Using the double fibration (11) a local section $s$ of $Q$ over $U \subset \mathscr{T}$ can be regarded as a section $\hat{s}$ of $p^{*} P$ over $\pi^{-1}(U)$ satisfying

$$
\left\langle\hat{s}^{*} A, X\right\rangle-i \hat{s}^{*} \Phi=0 \text {. }
$$


Here $A, \Phi$ are pulled back from $\mathbb{R}^{3}$ to $\mathbb{R}^{3} \times S^{2}$ and $X$ is the geodesic flow on $\mathbb{R}^{3}$ $\times S^{2}$.

Now if we pull back $A$ with $\hat{s}$ to a 1 -form on $\pi^{-1}(U)$ and take the $(0,1)$ component of the projection onto the orthogonal this defines a 1 -form $A^{0,1}$. If this 1 -form is invariant under the geodesic flow we can use it to define a $\bar{\partial}$-connection on $Q$. As the real sections span the holomorphic tangent space to any point of $\mathscr{T}$ it suffices to show

$$
\frac{\partial}{\partial t}\left[A\left(\frac{\partial}{\partial x}+i \frac{\partial}{\partial y}\right)\right]=0,
$$

or in the obvious notation $\partial_{t}\left(A_{x}+i A_{y}\right)=0$. Now from Eq. (19) we have $A_{t}=i \Phi$, and using the definition of curvature we obtain

$$
\begin{aligned}
\partial_{t}\left(A_{x}+i A_{y}\right) & =F_{t x}+\partial_{x} A_{t}-\left[A_{t}, A_{x}\right]+i\left\{-F_{y t}+\partial_{y} A_{t}+\left[A_{y}, A_{t}\right]\right\} \\
& =F_{t x}-\nabla_{y} \Phi+i\left\{-F_{y t}+\nabla_{x} \Phi\right\}=0
\end{aligned}
$$

because of the Bogomolny equations. Because $A$ is a connection on $\mathbb{R}^{3}$ the transformation properties (15) are clearly satisfied.

As in [9] we note that any $(0,2)$ form is a multiple of $\bar{u}_{1} \wedge \bar{u}_{2}$, where $u_{1}$ is in the direction of the fibre of $S^{2} \times \mathbb{R}^{3} \rightarrow \mathbb{R}^{3}$. But $A$ on $S^{2} \times \mathbb{R}^{3}$ is a pullback connection to it follows that the $\bar{\partial}$-curvature vanishes and that the bundle $Q$ has a holomorphic structure.

Clearly if we construct an associated vector bundle this $\bar{\partial}$-connection induces on it the $\bar{\partial}$-operator defined by Hitchin in [9].

To show triviality over real sections we choose some point $x \in \mathbb{R}^{3}$ and $p \in P$ in the fibre over $x$, then for each line $\gamma$ through $x$ we can choose a section $s: \gamma \rightarrow P$ with $s(x)=p$ and $\left\langle s^{*} A, \gamma_{*}\right\rangle-i s^{*} \Phi=0$. This defines a section $\hat{s}$ over $S^{2} \times \mathbb{R} \subset S^{2} \times \mathbb{R}^{3}$ but as $A$ is a pullback connection we must have $\left(\hat{s}^{*} A\right)^{0,1}=0$.

The construction of the real structure $\sigma$ follows from the real structure on $P^{c}$ and the fact that $\tau(\gamma)=-\gamma$.

The inverse construction is done by the same method used by Hitchin in [9] applied to the adjoint bundle.

The theorem is now proved.

As an aside we note that because the monopole equations are defined in the adjoint representation they should not depend on $\pi_{1}(K)$. To see this in the twistor picture assume we start with a group $G$ with universal cover $\tilde{G}$ and therefore have the exact sequence

$$
0 \rightarrow \pi_{1}(G) \rightarrow \tilde{G} \rightarrow G \rightarrow 0 .
$$

Using suitable transition functions for $Q$ we see that the obstruction to lifting it to a $\widetilde{G}$ bundle is a two cochain with values in $\pi_{1}(G)$ which is a cocycle because $\pi_{1}(G)$ is central in $\widetilde{G}$. Moreover $H^{2}\left(\mathscr{T}, \pi_{1}(G)\right)$ restricts isomorphically to $H^{2}\left(\mathbb{P}_{1}, \pi_{1}(G)\right)$ for any real section and $Q$ is holomorphically trivial on real sections so this obstruction must vanish. As any two lifts differ by an element of $H^{1}\left(\mathscr{T}, \pi_{1}(G)\right)=0$ the lift is unique and we may as well assume $\pi_{1}(G)=0$.

To complete the twistor correspondence we must consider the effect of the boundary conditions. In [9] Hitchin observed that for SU(2) they implied that the 
bundle $E \rightarrow \mathscr{T}$ has a sub-bundle which means that the $\operatorname{SL}(2, \mathbb{C})$ frame bundle of $E$ has a reduction to a Borel subgroup - the upper triangular matrices. We shall see that this is true in general where for the group $G$ a Borel subgroup is a maximal solvable subgroup $B C G$. It can be chosen so that $B \cap K=T$ the maximal torus defined by the Higgs field at infinity.

Before stating this result we recall the definition of the bundle $L^{\lambda}(\lambda \in \mathbb{R})$ defined by Hitchin in [9]. This is the bundle on $\mathscr{T}$ defined by a U(1) monopole with Higgs field $i \lambda$. Note also that if $R$ is a reduction of $Q$ to a Borel subgroup $B$ and $\lambda \in L T^{*}$ any weight of $K$ the induced representation $\lambda: B \rightarrow \mathbb{C}^{X}$ defines an associated line bundle $R(\lambda)$ consisting of equivalence classes $[r, z]$, of pairs $(r, z)$, where $(r, z)$ $\sim\left(r b, \lambda\left(b^{-1}\right) z\right)$ for all $b \in B$. The result we need is

Theorem (21). If $(A, \Phi)$ satisfies the BPS boundary conditions with reduction at infinity to a maximal torus $T$, then the holomorphic bundle $Q$ has two reductions $R^{+}$, $R^{-}$, to $B, \bar{B}$ sub-bundles respectively such that

(1) $\sigma\left(R^{+}\right)=R^{-}$.

(2) $R^{ \pm}(\lambda)=L^{\lambda\left(\Phi^{\infty}\right)}\left(\mp 2 \lambda\left({ }^{*} F^{\infty}\right)\right)$.

Proof. The essential point of the proof is to define a Borel sub-algebra bundle $X \subset$ ad $Q$ using a decay condition as in [9]. We recall that there Hitchin showed that for the twistor correspondence for vector bundles a local solution $s_{z}$ of the equation $\left(\nabla_{t}-i \Phi\right) S_{z}=0$ had the property that

$$
\left\|\bar{\partial} s_{z}\right\| \leqq \text { const }\left\|s_{z}\right\| \quad \text { as } t \rightarrow \pm \infty \text {. }
$$

Therefore to find a sub-bundle fixed by the $\bar{\partial}$-operator, that is a holomorphic subbundle, we must look for solutions constrained to grow no faster than some given rate.

The bundle ad $Q$ can be realized as $Q \times{ }_{G} L G$ or equivalently as

$$
\operatorname{ad} Q(\gamma)=\left\{s \in \Gamma\left(\gamma, \operatorname{ad} P^{c}\right) \mid\left\langle\nabla s, \gamma_{*}\right\rangle-i[\Phi, s]=0\right\},
$$

where $\operatorname{ad} P^{c}$ is the complexification of $\operatorname{ad} P$. With out conventions the Borel subgroup $B$ has the Lie algebra

$$
L B=L T^{c} \bigoplus_{\alpha>0} L_{\alpha},
$$

where $\alpha>0$ precisely when $\alpha\left(\Phi^{\infty}\right)>0$. Applying the result of Coppel [5] we have that for each $x_{\alpha} \in L_{\alpha}\left(L_{0}=L T^{c}\right)$ there is a solution $s_{\alpha} \in \operatorname{ad} Q(\gamma)$ with

$$
s_{\alpha}(t) t^{-\alpha\left({ }^{*} F^{\infty}\right) / 2} e^{\alpha\left(\Phi^{\infty}\right) t} \rightarrow x_{\alpha} \text { as } t \rightarrow+\infty .
$$

So if we define

$$
X(\gamma)=\left\{s \in \Gamma\left(\gamma, \operatorname{ad} P^{c}\right) \mid\|s\| \text { is bounded as } t \rightarrow+\infty\right\}
$$

we see that $X(\gamma)$ is the span of the $s_{\alpha}$ with $\alpha \geqq 0$. Moreover if we bracket two such solutions they decay faster and as the possible rates of decay are bounded below (that is the number of positive roots is finite) all iterated brackets must eventually vanish. This shows that $X(\gamma)$ is a solvable subalgebra of $\operatorname{ad} Q(\gamma)$ and as $\operatorname{dim} X(\gamma)$ $=\operatorname{dim} L B$ it follows that $X(\gamma)$ is a Borel subalgebra. The comments at the beginning of the proof imply that $X$ is a holomorphic sub-bundle of ad $Q$. The sub- 
bundle $X$ defines a reduction $R^{+} C Q$ with structure group $B$ and by looking at decay in the other direction we obtain another sub-bundle $R^{-}$. Property (1) follows easily.

The proof of property (2) is that used by Hitchin in [9]. We work with a monopole with Higgs field $\Phi-i \lambda\left(\Phi^{\infty}\right)$. If we look at the equation $\nabla s=0$ instead of $\nabla-i \Phi s=0$ then this also defines a bundle $M$ on $\mathscr{T}$ and by looking at the subbundle with $+i \lambda\left(\Phi^{\infty}\right)$ eigenvalue we obtain a sub-bundle $M_{1} \subset M$. The BPS boundary conditions are sufficient to show that $M_{1}$ is $C^{1}$ and has a $C^{2}$ connection.

The Bogomolny equations imply that on each real section the curvature of this connection is a multiple of the volume form. Because the real sections span the tangent space to $\mathscr{T}$ at any point we deduce that the curvature form on $\mathscr{T}$ is the pullback of the curvature form restricted to the zero section. Now from [1] we see that a $C^{2}$ connection defines a holomorphic structure, and because the curvature is pulled back we must have $M_{1} \cong \pi^{*} 0(p)$ for some $p$. In fact it is not hard to show that $M_{1} \simeq \pi^{*} O\left(-2 \lambda\left({ }^{*} F^{\infty}\right)\right)$.

Then by the same method used by Hitchin we can show that $R^{+}(\lambda) \otimes L^{-\lambda\left(\Phi^{\infty}\right)}$ $=M_{1}$ and the theorem is proved.

At this stage the spectral curve $S$ can be defined as a subset of $\mathscr{T}$. Each fibre of the bundle ad $Q$ contains two Borel subalgebras ad $R^{+}$and ad $R^{-}$. In general any two Borel subalgebras contain a Cartan subalgebra and generically it is their intersection. So we define $S$ to be the collection of $\gamma \in \mathscr{T}$ such that $\operatorname{dim}(\operatorname{ad} R(\gamma)$ $\left.\operatorname{nad} R^{-}(\gamma)\right)>\operatorname{rank} K$ and this is a closed subset of $\mathscr{T}$. Now the proof that solutions of $(\nabla-i \Phi) s=0$ have certain decay rates depends on relating solutions of

$$
\left[\frac{d}{d r}-i\left(\Phi^{\infty}-\frac{* F^{\infty}}{2 r}+O\left(\frac{1}{r^{2}}\right)\right)\right] s=0
$$

to solutions of the unperturbed equation

$$
\left[\frac{d}{d r}-i\left(\Phi^{\infty}-\frac{* F^{\infty}}{2 r}\right)\right] s=0 .
$$

Coppel [5] shows that for small enough values of the integral of the perturbation there is a bijection between bounded solutions of both these equations. Now as the perturbation is $O\left(\frac{1}{r^{2}}\right)$ it follows that there is some $R>0$ such that any line further than $R$ from the origin has the perturbation term suitably bounded. However, the unperturbed equation has only a $\operatorname{rank} K$ dimensional space of bounded solutions and therefore this is true also of the equation $(\nabla-i \Phi) s=0$. But the bounded solutions clearly from ad $R^{+}(\gamma) \cap \operatorname{ad} R^{-}(\gamma)$, so we have shown that $S$ is contained in a compact subset of $\mathscr{T}$. As $S$ is closed it then follows that

Corollary (22). The spectral curve $S$ is compact.

To see how these ideas work in practice we consider the case of SU(3). Here the Higgs field has the asymptotic expansion

$$
\Phi=i \operatorname{diag}\left(\mu_{1}, \mu_{2}, \mu_{3}\right)-\frac{i}{2 r} \operatorname{diag}\left(k_{1}, k_{2}, k_{3}\right)+O\left(\frac{1}{r^{2}}\right) .
$$


We define the projection map $x_{i}$ by $x_{i}\left(i \operatorname{diag}\left(\mu_{1}, \mu_{2}, \mu_{3}\right)\right)=\mu_{i}$. The Borel subgroup $B$ is the subgroup of $\operatorname{SL}(3, \mathbb{C})$ consisting of all upper triangular matrices. The associated vector bundle $E=Q\left(\mathbb{C}^{3}\right)$ therefore has two flags

$$
E_{1}^{ \pm} \subset E_{2}^{ \pm} \subset E
$$

induced by $R^{ \pm}$. An application of Theorem (21) tells us that

$$
\begin{gathered}
E_{1}^{+}=R^{+}\left(x_{1}\right)=L^{\mu_{1}}\left(-k_{1}\right), \\
E_{2}^{+} / E_{1}^{+}=R^{+}\left(x_{2}\right)=L^{\mu_{2}}\left(-k_{2}\right), \\
E / E_{2}^{+}=R^{+}\left(x_{3}\right)=L^{\mu_{3}}\left(-k_{3}\right),
\end{gathered}
$$

and

$$
\begin{gathered}
E_{i}^{-}=L^{\mu_{3}}\left(k_{3}\right), \\
E_{2}^{-} / E_{1}^{-}=L^{\mu_{2}}\left(k_{2}\right), \\
E / E_{2}^{-}=L^{\mu_{1}}\left(k_{1}\right) .
\end{gathered}
$$

The real structure $\sigma$ on $Q$ induces an antiholomorphic isomorphism $\sigma: E \rightarrow E^{*}$ covering $\tau$ on $\mathscr{T}$, and if for any $W \subset E^{*}$ we define $W^{\perp}$ by $W^{\perp}=\{v \in E \mid \omega(v)$ $=0 \forall \omega \in W\}$, then $\sigma\left(E_{1}^{+}\right)^{\perp}=E_{2}^{-}, \sigma\left(E_{2}^{+}\right)^{\perp}=E_{1}^{-}$.

\section{Spectral Data}

The spectral curve $S$ has been defined as a subset of $\mathscr{T}$. In this section it will be examined in more detail using two different approaches; the representation theory of $K$ and the cell decomposition of $K / T$. This will enable us to show that $S$ is the union of $r=\operatorname{rank} K$ real, algebraic curves $\left\{S_{1}, \ldots, S_{r}\right\}$ labelled by the simple roots $\left\{\alpha_{1}, \ldots, \alpha_{r}\right\}$ and to determine a decomposition $S_{i} \cap S_{j}=S_{i j} \cup S_{j i}$ whenever $i$ and $j$ are joined on the Dynkin diagram. These curves and the decomposition together constitute the spectral data of the monopole.

If $K \rightarrow G L(V)$ is a representation of $K$ with lowest weight $-\lambda$ then it extends to a holomorphic representation of $G$ and therefore determines an associated vector bundle

$$
E=Q \times{ }_{G} V .
$$

As a representation of the torus $V$ decomposes into a direct sum of weight spaces

$$
V=\bigoplus_{\mu \in \pi} V(\mu),
$$

where $\pi$ is the set of weights of $V$. Using the facts that

$$
L B=L T^{c} \bigoplus_{\alpha>0} L_{\alpha}, \quad L \bar{B}=L T^{c} \bigoplus_{\alpha<0} L_{\alpha},
$$

and $L_{\alpha} \cdot V(\mu) \subset V(\mu+\alpha)$, we see that $V(-\lambda)$ is $\bar{B}$-stable and $\bigoplus_{\mu>-\lambda} V(\mu)$ is $B$-stable. 
The vector bundle $E$ therefore has two sub-bundles defined using the reductions $R^{+}$and $R^{-}$, these are

$$
E^{+}=R^{+}\left(\bigoplus_{\mu>-\lambda} V(\mu)\right), \quad E^{-}=R^{-}(V(-\lambda)) .
$$

Here and throughout $R^{+}(X)=R^{+} \times{ }_{B} X, Q(X)=Q \times{ }_{G} X$ etc. As these subbundles are of complementary dimensions we can define $S_{\lambda}$, the spectral curve for the weight $\lambda$, to be the points where $E^{-} \subset E^{+}$or the divisor of

$$
\phi: E^{-} \rightarrow E / E^{+} \text {. }
$$

From Theorem (21) we see that $S_{\lambda}$ has degree $4 \lambda\left({ }^{*} F^{\infty}\right)$.

If the representation is chosen to be unitary we can use the real structure $\sigma: Q \rightarrow Q$ to define an anti-holomorphic isomorphism $\sigma: E \rightarrow E^{*},[q, v] \mapsto[\sigma(q)$, $\langle v,-\rangle]$, where $\langle$,$\rangle is the hermitian inner product on V$ and $[q, v]$ is the equivalence class of $(q, v)$ in $Q \times_{G} V$. As the spaces $V(-\lambda)$ and $\bigoplus_{\mu>-\lambda} V(\mu)$ are orthogonal it is easy to check that

$$
\sigma\left(E^{ \pm}\right)=\left(E^{\mp}\right)^{\perp} \subset E^{*}
$$

Now we can show that $S_{\lambda}$ is real, that is, $\tau\left(S_{\lambda}\right)=S_{\lambda}$. From (28) we have the commuting diagram

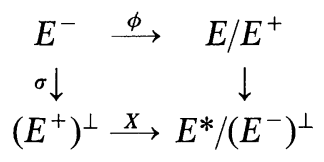

$(\operatorname{defining} X)$ where $\operatorname{div}(X)=\tau(\operatorname{div} \phi)=\tau\left(S_{\lambda}\right)$. Now $\operatorname{div} X=\operatorname{div} X^{*}$ and

$$
\left(E^{*} /\left(E^{-}\right)^{\perp}\right)^{*} \stackrel{X^{*}}{\longrightarrow}\left(E^{+}\right)^{\perp *}
$$

but $\left(E^{*} /\left(E^{-}\right)^{\perp}\right)^{*} \simeq E^{-},\left(E^{+}\right)^{\perp *} \simeq E / E^{+}$so we see that $X^{*}=\phi$. We have now nearly proved

Proposition (30). The spectral curve $S_{\lambda}$ is a real, compact, algebraic curve of degree $4 \lambda\left({ }^{*} F^{\infty}\right)$.

Proof. The only thing left to prove is compactness. But it is clear that if $\left(\operatorname{ad} R^{-}\right)$ $\cap\left(\operatorname{ad} R^{+}\right)$is a Cartan subalgebra then $E^{-} \cap E^{+}=\{0\}$, so $S_{\lambda} \subset S$ and therefore compact from (22).

The weights $\Lambda \subset L T^{*}$ form a lattice generated over $\mathbb{Z}$ by the fundamental dominant weights $\left\{\lambda_{1}, \ldots, \lambda_{r}\right\}$ satisfying

$$
\frac{2\left\langle\lambda_{i}, \alpha_{j}\right\rangle}{\left\langle\alpha_{j}, \alpha_{j}\right\rangle}=\delta_{i j} .
$$

For every dominant weight, that is every weight of the form $\sum_{i=1}^{r} m_{i} \lambda_{i}$ with nonnegative $m_{i}$, we have defined a spectral curve. Consider now two dominant weights $\mu_{1}$ and $\mu_{2}$ and let $\mu=\mu_{1}+\mu_{2}$. Denote the corresponding $K$ modules with lowest weights $-\mu,-\mu_{1}$ and $-\mu_{2}$ by $V, V_{1}$ and $V_{2}$. We wish to show that $S_{\mu}=S_{\mu_{1}}$ 
$+S_{\mu_{2}}$. If the consider the $K$ module $V_{1} \otimes V_{2}$ and take the irreducible component of this containing $V_{1}\left(-\mu_{1}\right) \otimes V_{2}\left(-\mu_{2}\right)$ this is just $V$. Now consider the map

$$
V \rightarrow V_{1} / \alpha>-\bigoplus_{\mu_{1}} V_{1}(\alpha) \otimes V_{2} / \bigoplus_{\alpha>-\mu_{2}} V_{2}(\alpha)
$$

which does not annihilate $V(-\mu)$. But this is a $\bar{B}$ map so it follows that its kernel is $\bigoplus_{\alpha>-\mu} V(\alpha)$. We therefore have a $\bar{B}$ isomorphism

$$
V / \bigoplus_{\alpha>-\mu} V(\alpha) \simeq \bigoplus_{i=1}^{2}\left(V / \bigoplus_{\alpha>-\mu_{i}} V_{i}(\alpha)\right)
$$

and a $B$ isomorphism

$$
V(-\mu) \simeq V_{1}\left(-\mu_{1}\right) \otimes V_{2}\left(-\mu_{2}\right)
$$

Forming associated bundles we obtain the commuting diagram

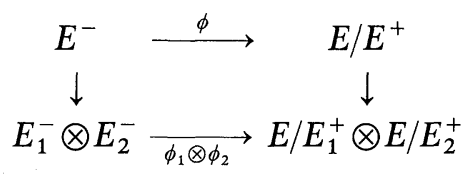

where $S_{\mu}=\operatorname{div} \phi, S_{\mu_{1}}=\operatorname{div} \phi_{1}$ and $S_{\mu_{2}}=\operatorname{div} \phi_{2}$. It follows that $S_{\mu}=S_{\mu_{1}}+S_{\mu_{2}}$.

We can now define the spectral curves $\left\{S_{1}, \ldots, S_{r}\right\}$ of the monopole to be $S_{i}$ $=S_{\lambda_{i}}$, and in view of the preceding remarks we have

Proposition (32). If $\lambda=\sum_{i=1}^{r} n_{i} \lambda_{i}$ is a dominant weight then

$$
S_{\lambda}=\sum_{i=1}^{r} n_{i} S_{i}
$$

We now see that $S$ contains the spectral curves $S_{i}$. Moreover $S$ is the zero set of the map

$$
\operatorname{det}\left(R^{-}\left(\bigoplus_{\alpha<0} L_{\alpha}\right)\right) \rightarrow \operatorname{det}\left(\operatorname{ad} Q / \operatorname{ad} R^{+}\right)
$$

so that $S=S_{2 \delta}$ where $2 \delta=\sum_{\alpha>0} \alpha=2 \sum_{i=1}^{r} \lambda_{i}$. It follows then from Proposition (32) that $S$ is the union of the $S_{i}$.

Now in the SU(2) case it was important that the line bundle $L^{2}$ restricted to the spectral curve $S$ had a section $\xi$. This section can be defined by the diagram

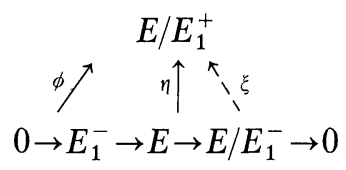


where the broken arrow indicates that $\xi$ is defined only where $\phi$ vanishes. More correctly we have the commuting diagram of sheaves

$$
\begin{aligned}
& 0 \rightarrow E_{1}^{-} \stackrel{\phi}{\rightarrow} E / E_{1}^{+} \rightarrow O_{S}\left(E / E_{1}^{+}\right) \rightarrow 0 \\
& \| \quad \uparrow \eta \quad \uparrow \xi \\
& 0 \rightarrow E_{1}^{-} \rightarrow E \quad \rightarrow \quad E / E_{1}^{-} \quad \rightarrow 0
\end{aligned}
$$

and here $\xi$ is a global sheaf map.

For the general case we can define a similar diagram and section $\xi_{i}$ and these will be important in the reconstruction of the monopole.

We note first that if $\lambda_{i}$ is a fundamental weight, then $V\left(-\lambda_{i}\right)$ is one dimensional, moreover $\lambda_{i}-\alpha_{i}=\lambda_{i}-\frac{2\left\langle\lambda_{i}, \alpha_{i}\right\rangle}{\left\langle\alpha_{i}, \alpha_{i}\right\rangle} \alpha_{i}$ is conjugate to $\lambda_{i}$ under the Weyl group so $V\left(-\lambda_{i}+\alpha_{i}\right)$ is also one dimensional. The space $V\left(-\lambda_{i}\right) \oplus V\left(-\lambda_{i}+\alpha_{i}\right)$ is stable under $\bar{B}$ so we can define the $i^{\text {th }}$ fundamental diagram

$$
\begin{aligned}
& R^{+}(V) / R^{+}\left(\underset{\mu>-\lambda_{\imath}}{\bigoplus_{i}} V(\mu)\right) \\
& \phi_{i} \uparrow_{\eta_{i}} \eta_{i} \\
& 0 \rightarrow R^{-}\left(V\left(-\lambda_{i}\right)\right) \rightarrow R^{-}\left(V\left(-\lambda_{i}\right) \oplus V\left(-\lambda_{1}+\alpha_{i}\right)\right) \rightarrow R^{-}\left(-\lambda_{i}+\alpha_{i}\right) \rightarrow 0 \text {. }
\end{aligned}
$$

Applying Theorem (21) and for simplicity not repeating the middle term we obtain

$$
\begin{aligned}
& L^{-\lambda_{i}\left(\Phi^{\infty}\right)}\left(m_{i}\right)
\end{aligned}
$$

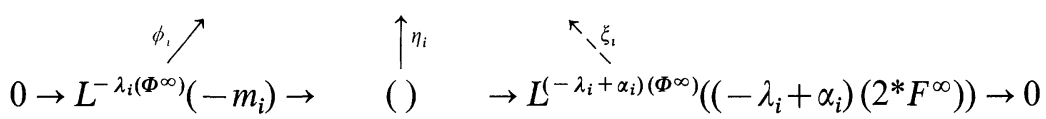

recalling that $\lambda_{i}\left(2 * F^{\infty}\right)=m_{i}(7)$. It follows that

$$
\xi_{i} \in H^{0}\left(S_{i}, L^{-\alpha_{i}\left(\Phi^{\infty}\right)}\left(2 m_{i}-\alpha_{i}\left(2^{*} F^{\infty}\right)\right)=H^{0}\left(S_{i}, L^{-\alpha_{i}\left(\Phi^{\infty}\right)}\left(\sum_{k \neq i} \frac{2\left\langle\alpha_{k}, \alpha_{i}\right\rangle}{\left\langle\alpha_{k}, \alpha_{k}\right\rangle} m_{k}\right)\right) .\right.
$$

As an example consider the SU(3) monopole. Here the spectral curves are

$$
S_{1}=\left\{\gamma \in \mathscr{T} \mid E_{1}^{+}(\gamma) \subset E_{2}^{-}(\gamma)\right\}, \quad S_{2}=\left\{\gamma \in \mathscr{T} \mid E_{1}^{-}(\gamma) \subset E_{2}^{+}(\gamma)\right\},
$$

and we observe that $S_{1} \cap S_{2}=S_{12} \cup S_{21}$, where

$$
S_{12}=\left\{\gamma \in \mathscr{T} \mid E_{2}^{+}(\gamma)=E_{2}^{-}(\gamma)\right\}, \quad S_{21}=\left\{\gamma \in \mathscr{T} \mid E_{1}^{+}(\gamma)=E_{1}^{-}(\gamma)\right\}
$$

In this case $\xi_{1}$ vanishes on $S_{21}$ and $\xi_{2}$ vanishes on $S_{12}$ precisely.

We can gain some insight into this decomposition by considering the flag bundle $F(E)$. For a three dimensional vector space $V$ a flag is a pair of subspaces $V_{1}$ $C V_{2}$ with $\operatorname{dim} V=i$. If we fix a flag $\left(V_{1}^{+}, V_{2}^{+}\right)$the set of all flags $F(V)$ has a nice cell 
decomposition given by

$$
\begin{aligned}
X_{0} & =\left\{\left(V_{1}^{-}, V_{2}^{-}\right) \mid\left(V_{i}^{-}\right) \text {is disjoint from }\left(V_{1}^{+}\right)\right\}, \\
X_{1} & =\left\{\left(V_{1}^{-}, V_{2}^{-}\right) \mid V_{1}^{+} \subset V_{2}^{-}, V_{1}^{+} \cap V_{2}^{-}=0, V_{2}^{-} \cap V_{2}^{+}=V_{1}^{+}\right\}, \\
X_{2} & =\left\{\left(V_{1}^{-}, V_{2}^{-}\right) \mid \text {as for } X_{1} \text { with } \pm \text { interchanged }\right\}, \\
X_{12} & =\left\{\left(V_{1}^{-}, V_{2}^{-}\right) \mid V_{2}^{-}=V_{2}^{+}, V_{1}^{+} \neq V_{1}^{-}\right\}, \\
X_{21} & =\left\{\left(V_{1}^{-}, V_{2}^{-}\right) \mid \text {as for } X_{12} \text { with } 1,2 \text { interchanged }\right\}, \\
X_{3} & =\left\{\left(V_{1}^{-}, V_{2}^{-}\right) \mid\left(V_{i}^{+}\right)=\left(V_{i}^{-}\right)\right\} .
\end{aligned}
$$

The natural ordering $X_{\alpha} \subset \bar{X}_{\beta}$ and the dimensions of these cells are

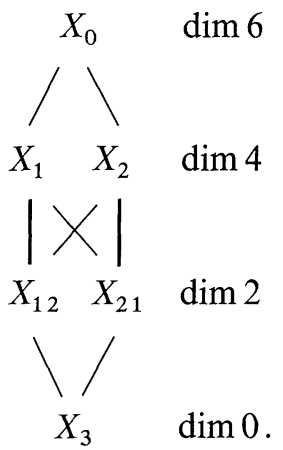

Now the flag bundle $F(E)$ is defined as $F(E)(\gamma)=F(E(\gamma))$. If we take the section defined by $\left(E_{i}^{-}\right)$this defines a cell decomposition of each fibre of $F(E)$ and intersecting this with $\left(E_{i}^{+}\right)$and projecting onto $\mathscr{T}$ yields $S_{1}, S_{2}, S_{12}$, and $S_{21}$. In the remainder of this section we shall generalize this example and show that whenever $i, j$ are joined on the Dynkin diagram there is a natural decomposition $S_{i}$ $\cap S_{j}=S_{i j} \cup S_{j i}$. Moreover the divisor of the sections $\xi_{i}$ can be defined in terms of the $S_{i j}$ and the Cartan matrix of the Lie algebra $L K$. The spectral curves and this division will be called the spectral data of the monopole. space

For a general group $G$ the flag manifold is the compact, complex homogeneous

$$
G / B \simeq K / T \text {. }
$$

The "opposite" Borel subgroup $\bar{B}$ acts naturally on the flag manifold $G / B$ which is a projective algebraic variety and the orbits of this action are known as the Bruhat cells. They are, in fact, algebraic affine spaces $\mathbb{C}^{m}$ of various dimensions and give a cell decomposition of $G / B$. The closures of these cells are algebraic (irreducible) subvarieties of $G / B$ that may have singularities.

The cells are naturally indexed by the Weyl group

$$
W=N(T) / T \rightarrow K / T \cong G / B .
$$

The natural partial ordering on the cells $c \leqq c^{\prime}$ if $c \subset \bar{c}^{\prime}$ induces a partial ordering on the Weyl group. This can be described as follows [3]. First we recall that $W$ is generated by the simple root reflections $\sigma_{i}=\sigma_{\alpha_{2}}$ and that for any $w \in W$ the minimal $r$ such that $\omega=\sigma_{i_{1}} \ldots \sigma_{i_{r}}$ is called $l(w)$ the length of $\omega$. Such a minimal expression for 
$\omega$ is called reduced. Then if $w$ is a reduced expression the set $S(w)$ of elements of $W$ obtained by deleting $\sigma_{i}$ 's from this expression depends only on $w$. The partial ordering on $W$ is defined by

$$
S(w)=\left\{w^{\prime} \in W \mid w^{\prime} \geqq w\right\} .
$$

The (real) dimension of any cell $X_{w}$ is then [3]

$$
\operatorname{dim} X_{w}=\operatorname{dim} G / B-2 l(w),
$$

and the cells define a basis for the homology $H_{*}(G / B ; \mathbb{Z})$.

The cohomology of $G / B$ can be realized as follows. Any weight $\lambda \in \Lambda \subset L T^{*}$ defines a representation $\lambda: B \rightarrow \mathbb{C}^{X}$ and therefore a holomorphic line bundle $\mathscr{L}^{\lambda}$ $=G \times{ }_{B} \mathbb{C}$, where $b(g, z)=\left(b g, \lambda(b)^{-1} z\right)$. Taking the Chern class of $\mathscr{L}^{-\lambda}$ defines a $\operatorname{map} \alpha: \Lambda \otimes \mathbb{Q} \rightarrow H^{2}(X, \mathbb{Q}), \lambda \mapsto c\left(\mathscr{L}^{-\lambda}\right)$, which extends to an algebra epimorphism $\alpha: R=S\left(\Lambda \otimes_{\mathbb{Z}} \mathbb{Q}\right) \rightarrow H^{*}(X, \mathbb{Q})$. The kernel of $\alpha$ is $J$ the ideal generated by the Weyl group invariant elements of positive degree.

The natural pairing of cohomology and homology is realized in [3] as a pairing between $R$ and the cells $\left\{X_{w}\right\}$. First we define for each $\sigma_{i}$ a map

$$
A_{i}: R \rightarrow R, \quad f \mapsto \frac{f-\sigma_{i}(f)}{\alpha_{i}},
$$

and then if $w \in W$ and $\sigma w=\sigma_{i_{1}} \ldots \sigma_{i_{r}}$ is reduced the map $A_{\sigma w}=A_{i_{1}} \ldots A_{i_{r}}$ depends only on $w$ and for $w \in W, f \in R$ the pairing is

$$
\left\langle X_{w}, f\right\rangle=\left(A_{w} f\right)(0) .
$$

Using this non-degenerate pairing we can define a dual basis $\left\{P_{w}\right\}$ of cohomology and in [3] it is also shown that if $\mathbb{P}$ denotes Poincare duality then

$$
\mathbb{P}\left(X_{w}\right)=P_{\text {ow }},
$$

where $\sigma$ is the unique longest element of $W$.

With these facts we can deduce a number of simple results.

Proposition (43) (i) $P_{\sigma \sigma_{k}}=\lambda_{k}$,

(ii) $\left\langle X_{\sigma \sigma_{i} \sigma_{j}}, \lambda_{k} \lambda_{l}\right\rangle=\delta_{i}^{k} \delta_{j}^{l}+\delta_{j}^{k} \delta_{i}^{l}-\delta_{k}^{j} \delta_{j}^{l} \frac{2\left\langle\alpha_{i}, \alpha_{j}\right\rangle}{\left\langle\alpha_{i}, \alpha_{i}\right\rangle}$,

(iii) $P_{i} P_{j}=P_{i j}+P_{j i}$,

(iv) $P_{i} P_{i}=\sum_{k \neq i} \frac{-2\left\langle\alpha_{k}, \alpha_{i}\right\rangle}{\left\langle\alpha_{k}, \alpha_{k}\right\rangle} P_{i k}$.

Proof. (i) If $l(w) \neq 1$ then $\left\langle P_{\sigma w}, \lambda_{k}\right\rangle=0$, moreover $\left\langle P_{\sigma \sigma_{i}, \lambda_{k}}\right\rangle=\frac{2\left\langle\alpha_{i}, \lambda_{k}\right\rangle}{\left\langle\alpha_{i}, \alpha_{i}\right\rangle}=\delta_{i}^{k}$.

(ii) $\left\langle X_{\sigma \sigma_{i} \sigma_{j}}, \lambda_{k} \lambda_{l}\right\rangle=A_{i} A_{j}\left(\lambda_{k} \lambda_{l}\right)=A_{i}\left\{\frac{\lambda_{k} \lambda_{l}-\sigma_{j}\left(\lambda_{k}\right) \sigma_{j}\left(\lambda_{l}\right)}{\alpha_{j}}\right\}$

$$
=A_{i}\left\{\lambda_{k} \delta_{j}^{l}+\lambda_{l} \delta_{j}^{k}-\delta_{k}^{j} \delta_{j}^{l} \alpha_{j}\right\}=\delta_{i}^{k} \delta_{j}^{l}+\delta_{l}^{k} \delta_{i}^{l}-\delta_{k}^{j} \delta_{j}^{l} \frac{2\left\langle\alpha_{i}, \alpha_{j}\right\rangle}{\left\langle\alpha_{i}, \alpha_{i}\right\rangle} .
$$


(iii) Let $P_{i} P_{j}=\sum_{k, l} m_{i j}^{k l} P_{k l}$. Then from (ii)

If $i \neq j$ then $\delta_{i}^{l} \delta_{i}^{j}=0$ so

$$
m_{i j}^{k l}=\left\langle P_{i} P_{j}, X_{\sigma \sigma_{k} \sigma_{l}}\right\rangle=\delta_{k}^{i} \delta_{l}^{j}+\delta_{l}^{i} \delta_{k}^{j}-\delta_{i}^{l} \delta_{l} \frac{2\left\langle\alpha_{k}, \alpha_{l}\right\rangle}{\left\langle\alpha_{k}, \alpha_{k}\right\rangle} .
$$

$$
P_{i} P_{j}=\sum_{k, l}\left(\delta_{k}^{i} \delta_{l}^{j} P_{k l}+\delta_{l}^{i} \delta_{k}^{j} P_{k l}\right)=P_{i j}+P_{j i} .
$$

(iv) If $i=j$ then because $k \neq l \delta_{k}^{i} \delta_{l}^{j}=\delta_{l}^{i} \delta_{k}^{j}=0$ so

$$
P_{i}^{2}=\sum_{k, l}-\delta_{i}^{l} \delta_{l}^{i} \frac{2\left\langle\alpha_{k}, \alpha_{l}\right\rangle}{\left\langle\alpha_{k}, \alpha_{k}\right\rangle} P_{i j}=\sum_{k \neq i} \frac{-2\left\langle\alpha_{k}, \alpha_{i}\right\rangle}{\left\langle\alpha_{k}, \alpha_{k}\right\rangle} P_{i k} .
$$

Now from the Borel-Weil theorem [16] we know that $H^{0}\left(G / B, \mathscr{L}^{\lambda}\right)$ is the representation of $G$ with lowest weight $\lambda$. Hence

$$
H^{0}\left(G / B, \mathscr{L}^{-\lambda_{i}}\right)=V\left(-\lambda_{i}\right) \oplus V\left(-\lambda_{i}+\alpha_{i}\right) \oplus \ldots,
$$

and $\operatorname{dim} V\left(-\lambda_{i}\right)=\operatorname{dim}\left(V\left(-\lambda_{i}+\alpha_{i}\right)\right)=1$. We then have

Proposition (44). (i) If $\xi \in V\left(\lambda_{i}\right) \operatorname{div}(\xi)=\bar{X}_{\sigma_{i}}$.

(ii) $\bar{X}_{\sigma_{i}} \cap \bar{X}_{\sigma_{j}}=\bar{X}_{\sigma_{i} \sigma_{j}} \cup \bar{X}_{\sigma_{j} \sigma_{i}}$ as varieties if $i \neq j$.

(iii) If $\xi \in V\left(\lambda_{i}\right)$ and $\eta \in V\left(\lambda_{i}+\alpha_{i}\right)$ then

$$
\operatorname{div}(\xi) \cap \operatorname{div}(\eta)=\sum_{k \neq l} \frac{-2\left\langle\alpha_{k}, \alpha_{i}\right\rangle}{\left\langle\alpha_{k}, \alpha_{k}\right\rangle} X_{\sigma_{k} \sigma_{1}} .
$$

Proof. (i) The Poincaré dual to $X_{\sigma_{i}}$ is from (42) $\mathbb{P}\left(X_{\sigma_{i}}\right)=P_{\sigma \sigma_{t}}=\lambda_{i}$. Hence $\operatorname{div}(\xi)$ is homologous to $\bar{X}_{\sigma_{i}}$. However, $\operatorname{div}(\xi)$ is stable under $\bar{B}$ so must be a union of cells. It follows that $\operatorname{div}(\xi)=\bar{X}_{\sigma_{i}}$.

(ii) Consider the set theoretic intersection $\bar{X}_{\sigma_{i}} \cap \bar{X}_{\sigma_{j}}$. This is fixed under $\bar{B}$ and therefore is a union of cells. Now clearly $\sigma_{j} \sigma_{i}, \sigma_{i} \sigma_{j} \leqq \sigma_{i}$ and $\sigma_{j} \sigma_{i}, \sigma_{i} \sigma_{j} \leqq \sigma_{j}$ so

$$
\bar{X}_{\sigma_{i}} \cap \bar{X}_{\sigma_{J}} \supset \bar{X}_{\sigma_{i} \sigma_{j}} \cup \bar{X}_{\sigma_{j} \sigma_{i}} .
$$

On the other hand if $X_{w} \subset \bar{X}_{\sigma_{i}} \cap \bar{X}_{\sigma_{j}}$, then $w<\sigma_{i}, \sigma_{j}$, so a reduced expression of $\sigma w$ contains $\sigma_{i}$ and $\sigma_{j}$. Therefore either $\sigma_{i} \sigma_{j}>w$ or $\sigma_{j} \sigma_{i}>w$ in either case $X_{w} \subseteq \bar{X}_{\sigma_{i} \sigma_{j}}$ $\cup \bar{X}_{\sigma_{j} \sigma_{\imath}}$, and it follows that

$$
\bar{X}_{\sigma_{i}} \cap \bar{X}_{\sigma_{j}}=\bar{X}_{\sigma_{i} \sigma_{j}} \cup \bar{X}_{\sigma_{j} \sigma_{i}} .
$$

Now we have to check for multiplicities in this formula. From [8] we see that this can be done by knowing the expansion of $\bar{X}_{\sigma_{i}} \cdot \bar{X}_{\sigma_{j}}$ in homology. As intersection pairing is Poincare dual to cup product we can expand $\mathbb{P}\left(\bar{X}_{\sigma_{i}} \cdot \bar{X}_{\sigma_{t}}\right)$ $=\mathbb{P}\left(X_{\sigma_{i}}\right) \mathbb{P}\left(X_{\sigma_{J}}\right)=\lambda_{i} \lambda_{j}$ in terms of $\mathbb{P}\left(\bar{X}_{\sigma_{k} \sigma_{l}}\right)=P_{\sigma \sigma_{k} \sigma_{l}}$. So we wish to determine the $m_{i j}^{h l}$ in

$$
\lambda_{i} \lambda_{j}=\sum m_{i j}^{k l} p_{\sigma \sigma_{k} \sigma_{l}} .
$$

But from (ii) $m_{i j}^{k l}=\left\langle x_{\sigma \sigma_{k} \sigma_{l}}, \lambda_{i j}\right\rangle$, so because $i \neq j$ we have

$$
\bar{X}_{\sigma_{l}} \cap \bar{X}_{\sigma_{i}}=\bar{X}_{\sigma_{i} \sigma_{J}}+\bar{X}_{\sigma_{j} \sigma_{i}} .
$$
(iii) This is proved as for (ii) but this time using $P_{i}^{2}=\sum_{k \neq i} \frac{-2\left\langle\alpha_{k}, \alpha_{i}\right\rangle}{\left\langle\alpha_{i}, \alpha_{i}\right\rangle} P_{k i}$ from
Proposition (43). $\quad \square$ 
We now return to the spectral curves. The bundle $Q$ has two reductions $R^{+}$, $R^{-}$to $B$ and $\bar{B}$ sub-bundles. Because $G$ acts on $G / B$ we can form the associated fibration

$$
Q(G / B) \simeq R^{+}(G / B) \simeq R^{-}(G / B) .
$$

Now the bundle $R^{+}(G / B)$ has a tautological section $R^{+}$and the fibres of $R^{-}(G / B)$ have a cell decomposition given by the actions of $\bar{B}$. We can now define

and we have

$$
T_{i}=\left(R^{+}\right)^{*} R^{-}\left(\bar{X}_{\sigma_{t}}\right),
$$

Proposition (47). The curve $T_{i}$ is the $i^{\text {th }}$ spectral curve $S_{i}$.

Proof. From the Borel-Weil theorem the representation with lowest weight $-\lambda_{i}$ is

$$
V=H^{0}\left(G / B, \mathscr{L}^{-\lambda_{i}}\right)=V\left(-\lambda_{i}\right) \oplus V\left(-\lambda_{i}+\alpha_{i}\right) \oplus \ldots
$$

Consider the evaluation map

$$
G / B \times V\left(-\lambda_{i}\right) \rightarrow \mathscr{L}^{-\lambda_{i}} .
$$

This is, in fact, invariant under $\bar{B}$ and from Proposition (44) it vanishes on $\bar{X}_{\sigma_{1}}$. It follows that $R^{-}\left(\bar{X}_{\sigma_{i}}\right)$ is defined by the vanishing of $R^{-}\left(G / B \times V\left(-\lambda_{i}\right)\right)$ $\rightarrow R^{-}\left(\mathscr{L}^{-\lambda_{i}}\right)$. So $T_{i}$ is the divisor of

$$
R^{+*}\left(R^{-}\left(G / B \times V\left(-\lambda_{i}\right)\right)\right) \rightarrow R^{+*} R^{-}\left(\mathscr{L}^{-\lambda_{i}}\right) .
$$

Now on each fibre of $R^{-}(G / B) \rightarrow T \mathbb{P}_{1}$ the bundle $R^{-}\left(G / B \times V\left(-\lambda_{i}\right)\right)$ is a copy of $V\left(-\lambda_{i}\right)$, and therefore we have

$$
R^{+*}\left(R^{-}\left(G / B \times V\left(-\lambda_{i}\right)\right)=R^{-}\left(V\left(-\lambda_{i}\right)\right) .\right.
$$

To determine the second bundle consider the evaluation map

$$
G / B \times V \rightarrow \mathscr{L}^{-\lambda_{i}} .
$$

This is also $\bar{B}$ invariant so defines

$$
R^{+}(V) \rightarrow\left(R^{+}\right) * R^{-}(G / B \times V) \rightarrow\left(R^{+}\right) * R^{-}\left(\mathscr{L}^{-\lambda_{\imath}}\right) .
$$

Taking an equivalence class $\left[r^{+}, \varphi\right]$ where $r^{+} \in R^{+}$and $\varphi \in H^{0}\left(G / B, \mathscr{L}^{-\lambda_{r}}\right)$, and following through this map we see that it vanishes when $\varphi(B)$ vanishes. Now the subspace of $\varphi \in H^{0}\left(G / B, \mathscr{L}^{-\lambda_{i}}\right)$ vanishing at $B$ has codimension 1 and is $B$ stable so must be

$$
\bigoplus_{\mu>-\lambda_{r}} V(\mu) \text {. }
$$

It is easy to check now that $T_{i}$ is the divisor of the natural map

$$
R^{-}\left(V\left(-\lambda_{i}\right)\right) \rightarrow R^{+}(V) / R^{+}\left({ }_{\mu>-\lambda_{l}} V(\mu)\right),
$$

and comparing with (27) we see that $T_{i}$ is the $i^{\text {th }}$ spectral curve.

Using this cell decomposition we can also define

$$
S_{i j}=\left(R^{+}\right) * R^{-}\left(\bar{X}_{\sigma_{i} \sigma_{j}}\right)
$$


and from Proposition (44) we deduce

Proposition (49). (i) $S_{i} \cap S_{j}=S_{i j} \cup S_{j i}$.

(ii) $\operatorname{div}\left(\xi_{i}\right)=\sum_{k \neq i} \frac{-2\left\langle\alpha_{k}, \alpha_{i}\right\rangle}{\left\langle\alpha_{k}, \alpha_{k}\right\rangle} S_{k i}$.

Proof. The result (i) is clear. For (ii) we inspect the proof of Proposition (47) and see that $\xi_{i}$ is the pullback by $R^{+}$of the map induced by $G / B \times V\left(-\lambda_{i}+\alpha_{i}\right) \rightarrow \mathscr{L}^{-\lambda_{i}}$ and from Proposition (44) the result follows.

To summarize; we have defined for any monopole some spectral data, this is a collection of curves $\left\{S_{1}, \ldots, S_{r}\right\}$ with a division $S_{i} \cap S_{j}=S_{i j} \cup S_{j i}$ when $i$ and $j$ are joined on the Dynkin diagram and from (35) and Proposition (49) we see that these are constrained by the requirement that the divisor

$$
\sum_{i \neq k} \frac{-2\left\langle\alpha_{k}, \alpha_{i}\right\rangle}{\left\langle\alpha_{k}, \alpha_{k}\right\rangle} S_{k i}
$$

represent the point on the Jacobian of $S_{i}$ defined by the line bundle

$$
L^{-\alpha_{i}\left(\Phi^{\infty}\right)}\left(\frac{-2\left\langle\alpha_{h}, \alpha_{i}\right\rangle}{\left\langle\alpha_{k}, \alpha_{k}\right\rangle}\right) S_{k i} .
$$

If we assume the curves are distinct and smooth we can do a naive parameter count. It is not difficult to show that $\tau\left(S_{i j}\right)=S_{j i}$ and the $S_{i}$ are real so it suffices to count complex parameters.

If $S_{i} \neq \emptyset\left(m_{i} \neq 0\right)$ we see from [9] that this determines $\left\{\left(m_{i}+1\right)^{2}-1\right\}-\left(m_{i}-1\right)^{2}$ parameters because $\operatorname{dim} H^{0}\left(\mathscr{T}, 0\left(2 m_{i}\right)\right)=\left(m_{i}+1\right)^{2}$ and $\operatorname{dim}$ Jacobian $S_{i}=\left(m_{i}-1\right)^{2}$. If $m=0$ this gives -1 so the parameter count is

$$
4 \sum_{i=1}^{r} m_{i}-\operatorname{rank} K+\operatorname{card}\left\{i \mid m_{i}=0\right\}
$$

agreeing with the result calculated by Weinberg in [16].

We have now constructed a map from the moduli space of all monopoles to a certain subvariety of the moduli space of curves and at smooth points these have the same dimension. In the next section we show that for a general $\mathrm{SU}(n), \operatorname{Sp}(n)$ or $\mathrm{SO}(2 n)$ monopole this map is one to one.

\section{Reconstructing the Monopole}

In this section we will show that the spectral data determines the general monopole for the classical groups $\mathrm{SU}(n), \mathrm{Sp}(n)$, and $\mathrm{SO}(2 n)$.

For each classical group we can form a standard associated bundle $E$ with a flag, real structure and some algebraic structure such as a skew or symmetric form. Each of these vector bundles has a number of exact sequences associated with its flag and by analyzing the representation theory we can show that these are "lifts" of the fundamental exact sequences in (33). To perform this lift we need to assume that $S_{i} \cap S_{j}$ is finite. In Proposition (53) we show that this is true for the general monopole, that is, there is a non-empty open subset of the moduli space of monopoles for which $S_{i} \cap S_{j}$ is finite. 
Having recovered the bundle we need to define the real structure and any algebraic structure so we can recover the principal bundle $Q$ with real structure as a sub-bundle of the frame bundle of $E$. However, the bundle $E$ came from a monopole so we know it admits the required structure. If the bundle $E$ has a nontrivial holomorphic automorphism the general twistor correspondence for linear equations implies that the bundle $\widetilde{E}$ on $\mathbb{R}^{3}$ has a non-trivial automorphism $s$ such that $d_{A} s=0$ and $[\Phi, s]=0$. It follows from this that $\left[F_{A}, s\right]=0$ and $\left[d_{A} \Phi, s\right]=0$. Now $\operatorname{ad} P^{c} \rightarrow \operatorname{End}(\widetilde{E})$ by an irreducible representation $L K \rightarrow \operatorname{End}(V)$ where $\widetilde{E}=P(V)$. It follows from Schurs lemma that if $\left[s, \operatorname{ad} P^{c}\right]=0$ then $s$ is a scalar. Now if the connection is irreducible the values of the curvature $\operatorname{span} \operatorname{ad} P^{c}$, so we have proved

Proposition (51). If $(A, \Phi)$ is an irreducible monopole and $V$ an irreducible representation, then $Q(V)$ has no non-trivial automorphisms.

It follows from this proposition that if we start with two monopoles $(A, \Phi)$, $\left(A^{\prime}, \Phi^{\prime}\right)$ for classical groups and produce two vector bundles $Q(V), Q\left(V^{\prime}\right)$ on $\mathscr{T}$ which are isomorphic as vector bundles then they are isomorphic as vector bundles with real and algebraic structure. This means that to show that the spectral data determines the monopole it suffices to show it determines the vector bundle.

We show now that there is a non-empty open subset of the moduli space of monopoles for which $S_{i} \cap S_{j}$ is finite. As this condition is "open" we only have to show one such monopole exists. We observe first that because the spectral curves $\left\{S_{1}, \ldots, S_{r}\right\}$ are compact there exist closed balls $\left\{D_{1}, \ldots, D_{r}\right\}$ in $\mathbb{R}^{3}$ with the property that any line on $S_{i}$ must intersect $D_{i}$. Then we deduce

Lemma (52). If $D_{i} \cap D_{j}=\emptyset$, then $S_{i} \cap S_{j}$ is finite.

Proof. If $D_{i} \cap D_{j}=\emptyset$ then there are an infinite number of points $x \in \mathbb{R}^{3}$ such that no line through $x$ goes through both $D_{i}$ and $D_{j}$. This means the real section $p_{x}$ does not intersect $S_{i} \cap S_{j}$. But if $S_{i} \cap S_{j}$ is not finite it contains an algebraic curve that must intersect every real section so the result is proved.

Proposition (53). The general monopole has $S_{i} \cap S_{j}$ finite $\forall i \neq j$.

Proof. We use Theorem II of [14] which says that if we choose SU(2) solutions $\left(A^{i}, \Phi^{i}\right), i=1, \ldots, r$, a number $d$ large enough and points $\left\{x_{1}, \ldots, x_{r}\right\}$ with $\mid x_{i}$ $-x_{j} \mid>d$, then there is a $K$ monopole solution $(A, \Phi)$ such that on some ball of radius $R(d)$ about $x_{i}$ it satisfies

$$
\left|A-g^{-1} A^{i} g-g^{-1} d g\right|^{2}+\left|\Phi-g^{-1} \Phi^{i} g\right|^{2}<\varepsilon(d),
$$

where $g$ is a gauge transformation defined on his ball. Moreover as $d \rightarrow \infty, R(d)$ gets larger and $\varepsilon(d)$ gets smaller. Also if the topological charge of $\left(A^{i}, \Phi^{i}\right)$ is $m_{i}$ then the topological charges of $(A, \Phi)$ are $\left\{m_{1}, \ldots, m_{r}\right\}$.

Now for each of $\left(A^{i}, \Phi^{i}\right)$ there is a ball $B^{i}$ intersecting all the lines on the spectral curve of $\left(A^{i}, \Phi^{i}\right)$ so there is a $d$ such that

$$
B^{i} C B\left(x_{i}, R(d)\right) \text {. }
$$

From the inequality between $\left(A^{i}, \Phi^{i}\right)$ and $(A, \Phi)$ it follows that $D_{i}$ (as defined above for $(A, \Phi))$ is "close" to $B^{i}$. In particular, if we choose $d$ large enough we must have 
$D_{i} \subset B\left(x_{i}, R(d)\right)$. From the method of proof and the fact that the topological charge of $\left(A^{i}, \Phi^{i}\right)$ is $m_{i}$ there cannot be other lines in $S_{i}$ not intersecting $D_{i}$.

The result now follows from the lemma.

We now prove the basic lemma needed for the reconstruction. Let $I, J$ be line bundles and $E, F$ vector bundles over a complex manifold. Assume the following diagram commutes where the horizontal sequence is exact.

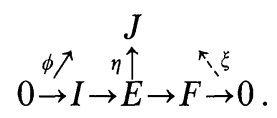

We use the dashed arrow to indicate that $\xi$ is only defined on $S$, the divisor of $\phi$. Then from the ideal exact sheaf sequence of $\phi$ we have

$$
0 \rightarrow F^{*} \otimes I \stackrel{\phi}{\rightarrow} F^{*} \otimes J \rightarrow O_{S}\left(J \otimes F^{*}\right) \rightarrow 0,
$$

and using the exact cohomology sequence we have the coboundary map

$$
H^{0}\left(O_{S}\left(J \otimes F^{*}\right)\right) \stackrel{\delta}{\rightarrow} H^{1}\left(F^{*} \otimes I\right) .
$$

Now $H^{1}\left(F^{*} \otimes I\right)$ classifies (isomorphism classes of extensions of $I$ by $F$ and we have Lemma (57). In the situation above $\delta(\xi)=[E]$.

Proof. We note that from an extension

$$
0 \rightarrow I \rightarrow E \rightarrow F \rightarrow 0,
$$

we can form the exact sequence

$$
0 \rightarrow \operatorname{Hom}(F, I) \rightarrow \operatorname{Hom}(F, E) \rightarrow \operatorname{Hom}(F, F) \rightarrow 0,
$$

and the exact cohomology sequence of this has the piece

$$
H^{0}(\operatorname{Hom}(F, F)) \stackrel{\delta}{\rightarrow} H^{1}(\operatorname{Hom}(F, I)) .
$$

Then if id $\in H^{0}(\operatorname{Hom}(F, F))$ is the identity map $\delta(\mathrm{id})=[E]$ is the equivalence class of the extension

$$
0 \rightarrow I \rightarrow E \rightarrow F \rightarrow 0 .
$$

Now as $F$ as a locally free sheaf [that is $O(F)$ the sheaf of holomorphic sections of $F$ is a locally free sheaf] the covariant functor $\operatorname{Hom}(F,-)$ is exact. The diagram (54) can be written as

$$
\begin{aligned}
& 0 \rightarrow I \stackrel{\phi}{\rightarrow} J \rightarrow O_{S}(J) \rightarrow 0
\end{aligned}
$$

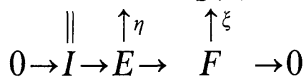

where both horizontal sequences of sheaves are exact and $\xi$ is a globally defined sheaf map. Applying the functor $\operatorname{Hom}(F,-)$ to this diagram we obtain

$$
\begin{gathered}
0 \rightarrow \operatorname{Hom}(F, I) \stackrel{\phi_{*}}{\rightarrow} \operatorname{Hom}(F, J) \rightarrow \operatorname{Hom}\left(F, O_{S}(J)\right) \rightarrow 0 \\
\uparrow \eta_{*} \\
0 \rightarrow \operatorname{Hom}(F, I) \rightarrow \operatorname{Hom}(F, E) \rightarrow \operatorname{Hom}(F, F) \rightarrow 0
\end{gathered}
$$

and clearly $\xi_{*}(\mathrm{id})=\xi_{o} \mathrm{id}=\xi$. 
Now using the exact cohomology sequence we have the commuting diagram

$$
\begin{gathered}
H^{0}\left(\operatorname{Hom}\left(F, O_{S}(J)\right)\right. \\
\uparrow_{*} \\
H^{0}(\operatorname{Hom}(F, F))
\end{gathered} \overbrace{}^{\delta} H^{1}(\operatorname{Hom}(F, I))
$$

and therefore $\delta(\xi)=\delta\left(\xi_{*} \mathrm{id}\right)=\delta^{\prime}(\mathrm{id})=[E]$ as required.

We note that the dashed arrow must be carefully interpreted when $S$ is not irreducible. The correct interpretation is to regard $\xi$ as a global sheaf map - as we have in this proof.

We will now prove the following theorem by working through each of the classical groups $\mathrm{SU}(n), \mathrm{Sp}(n), \mathrm{SO}(2 n)$ separately.

Theorem (58). The general irreducible monopole for the groups $\mathrm{SU}(n), \mathrm{Sp}(n)$, and $\mathrm{SO}(2 n)$ is determined by its spectral data.

The Lie algebra of SU( $n)$ is the collection of all $n \times n$ complex matrices $X$ with $X=-X^{*}$ and $\operatorname{tr} X=0$. For the torus of $L S U(n)$ we can take the diagonal matrices $i \operatorname{diag}\left(a_{1}, \ldots, a_{n}\right)$ with $a_{i} \in \mathbb{R}$ and $\sum_{i=1}^{m} a_{i}=0$. If we choose the fundamental Weyl chamber to satisfy $a_{1}>\ldots>a_{n}$, then the asymptotic expansion of the Higgs field is

$$
\Phi=i \operatorname{diag}\left(\mu_{1}, \ldots, \mu_{n}\right)-\frac{i}{2 r} \operatorname{diag}\left(k_{1}, \ldots, k_{n}\right)+O\left(\frac{1}{r^{2}}\right),
$$

where $\mu_{1}>\ldots>\mu_{n}$ and $\sum \mu_{i}=\sum k_{i}=0$. If $x_{j}$ is the projection $x_{j}\left(i \operatorname{diag}\left(a_{1}, \ldots, a_{n}\right)\right)$ $=a_{j}$ then the positive roots are $\left\{x_{i}-x_{j} \mid 1 \leqq i<j \leqq n\right\}$ and the simple roots are $\left\{x_{1}\right.$ $\left.-x_{2}, \ldots, x_{n-1}-x_{n}\right\}$. Then the fundamental representations are $\mathbb{C}^{n}$, $\Lambda^{2} \mathbb{C}^{n}, \ldots, \Lambda^{n-1} \mathbb{C}^{n}$ with highest weights $\left\{x_{1}, x_{1}+x_{2}, \ldots, x_{1}+x_{2}+\ldots+x_{n-1}\right\}$. So from (7) we see that the topological charges are

$$
m_{1}=k_{1}, \quad m_{2}=k_{1}+k_{2}, \ldots, m_{n-1}=k_{1}+\ldots+k_{n-1} .
$$

The Dynkin diagram of $\mathrm{SU}(n)$ is

$$
\begin{array}{lllll}
0 & 0 & 0 & - & -0 \\
1 & 2 & 3 & \ldots & n-1 .
\end{array}
$$

The standard representation fo $\mathrm{SU}(n)$ is $\mathbb{C}^{n}$ and this has weights $\left\{x_{1}, \ldots, x_{n}\right\}$ with highest weight $x_{1}$. As $x_{1}=x_{i-1}+\left(x_{i}-x_{i-1}\right)$ the weights are ordered by

$$
x_{1}>x_{2}>\ldots>x_{n} \text {. }
$$

The weight decomposition of $\mathbb{C}^{n}$ is just the standard decomposition $\mathbb{C}^{n}$ $=\mathbb{C} \oplus \ldots \oplus \mathbb{C}$ and the Borel subgroup (the upper triangular matrices) fixes the standard flag

$$
\mathbb{C} \subset \mathbb{C} \oplus \mathbb{C} \subset \ldots \mathbb{C} V \ldots \oplus \mathbb{C}=\mathbb{C}^{n}
$$

If we have an $\mathrm{SU}(n)$ monopole the twistor correspondence defines an $\operatorname{SL}(n, \mathbb{C})$ bundle $Q$ and two reductions, $R^{+}$and $R^{-}$, to Borel sub-bundles. The Borel 
subgroups $B$ and $\bar{B}$ are the upper and lower triangular matrices and if $X \in \operatorname{SL}(n, \mathbb{C})$ then $\bar{X}=\left(X^{*}\right)^{-1}$.

If we form the associated vector bundle $E=Q\left(\mathbb{C}^{n}\right)$ this is naturally isomorphic to $R^{+}\left(\mathbb{C}^{n}\right)$ and $R^{-}\left(\mathbb{C}^{n}\right)$ and the latter contain the flags

$$
\left\{\begin{array}{l}
E_{i}^{+}=R^{+}\left(\mathbb{C}_{i}^{+}\right) \\
E_{i}^{-}=R^{-}\left(\mathbb{C}_{i}^{-}\right),
\end{array}\right.
$$

where $\left\{\mathbb{C}_{i}^{ \pm}\right\}$are the flags

$$
\begin{gathered}
\mathbb{C}_{i}^{+}=\left\{\left(z_{1}, \ldots, z_{i}, 0, \ldots, 0\right)^{t} \mid z_{i} \in \mathbb{C}\right\} \subset \mathbb{C}^{n}, \\
\mathbb{C}_{i}^{-}=\left\{\left(0, \ldots, z_{n-i+1}, \ldots, z_{n}\right)^{t} \mid z_{i} \in \mathbb{C}\right\} \subset \mathbb{C}^{n} .
\end{gathered}
$$

As $\operatorname{SL}(n, \mathbb{C})$ is acting on the left of $\mathbb{C}^{n}$ these are stabilized by the upper and lower triangular matrices respectively.

The spectral curve $S_{n-p}$ is the set of $\gamma \in \mathscr{T}$ at which $E_{p}^{-}(\gamma) \cap E_{n-p}^{+}(\gamma) \neq 0$. This follows from the fact that the representation with lowest weight $-x_{1}-x_{2}$ $-\ldots-x_{n-p}=x_{n-p+1}+\ldots+x_{p}$ is $\Lambda^{p} \mathbb{C}^{n}$ so $S_{n-p}$ is defined by

$$
\phi_{p}: \Lambda^{p}\left(E_{p}^{-}\right) \rightarrow \Lambda^{p}\left(E / E_{n-p}^{+}\right) \text {. }
$$

From Theorem (21) we know that

$$
R^{ \pm}(\lambda) \cong L^{\lambda\left(\Phi^{\infty}\right)}\left( \pm 2 \lambda\left({ }^{*} F^{\infty}\right)\right)
$$

and applying this to the quotients of the flags above we obtain the exact sequences

$$
\begin{aligned}
& \begin{aligned}
& E_{1}^{+} \stackrel{\sim}{\rightarrow} L^{\mu_{1}}\left(-k_{1}\right) \\
0 \rightarrow E_{1}^{+} \rightarrow & E_{2}^{+} \rightarrow L^{\mu_{2}}\left(-k_{2}\right) \rightarrow 0
\end{aligned} \\
& 0 \rightarrow E_{n-1}^{+} \rightarrow E \rightarrow L^{\mu_{n}}\left(-k_{n}\right) \rightarrow 0 \\
& E_{1}^{-} \leadsto L^{\mu_{n}}\left(k_{n}\right) \\
& 0 \rightarrow E_{1}^{-} \rightarrow E_{2}^{-} \rightarrow L^{\mu_{n-1}}\left(k_{n-1}\right) \rightarrow 0 \\
& 0 \rightarrow E_{n-1}^{-} \rightarrow \dot{E} \rightarrow L^{\mu_{1}}\left(k_{1}\right) \quad \rightarrow 0
\end{aligned}
$$

Each of the exact sequences in (67) defines a diagram

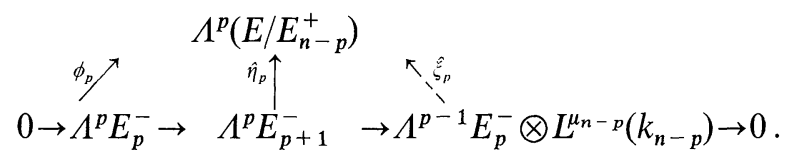

and if we join two of these together we obtain a diagram

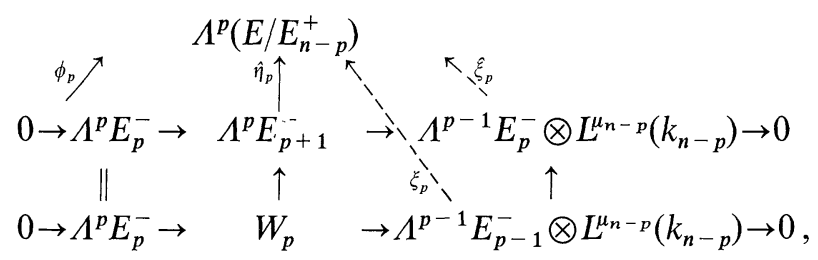


where $W_{p}$ is the appropriate bundle that makes the lower exact sequence the pullback of the upper. Some representation theory shows that the lower exact sequence is the $p^{\text {th }}$ fundamental exact sequence (33). In particular the divisor of $\xi_{p}$ is determined by the spectral data.

Now we see from Lemma (57) that the spectral curves and the sections $\xi_{i}$ are sufficient to recover the bundle $E$. This is done by an iterative procedure along the flag and the observation that

$$
\Lambda^{p} E_{p+1}^{-} \cong\left(E_{p+1}^{-}\right)^{*} \otimes \operatorname{det}\left(E_{p+1}^{-}\right) \cong\left(E_{p+1}^{-}\right)^{*} \otimes L^{\mu_{n-p}+\ldots+\mu_{n}}\left(k_{n-p}+\ldots+k_{n}\right) .
$$

On the other hand the spectral data determines the spectral curves and the sections $\xi_{i}$. We now have only to show that $\left\{S_{i}, \xi_{i}\right\}$ can be determined from $\left\{S_{i}, \hat{\xi}_{i}\right\}$.

We first observe that if we have recovered the exact sequence of the diagram (69) then $\phi_{p}$ is given as the map defining $S_{n-p}$ and $\hat{\eta}_{p}$ is unique. The latter fact is established by taking two such $\hat{\eta}_{p}$ and projecting their difference to obtain a global map

$$
\Lambda^{p-1} E_{p}^{-} \otimes L^{\mu_{n-p}}\left(k_{n-p}\right) \rightarrow \Lambda^{p}\left(E / E_{n-p}^{+}\right) .
$$

Then using the various exact sequences and the following lemma we can show this difference must vanish

Lemma (70) For any $\mu \in \mathbb{R}, \mu \neq 0$ and $k \in \mathbb{Z}, H^{0}\left(\mathscr{T}, L^{\mu}(k)\right)=0$.

Proof [11].

Now $\hat{\eta}_{p-1}$ can be used to define $\xi_{p}$ given $\xi_{p}$ because we have

Lemma (71). On the set $S_{n-p}-S_{n-p+1}$ the following diagram commutes

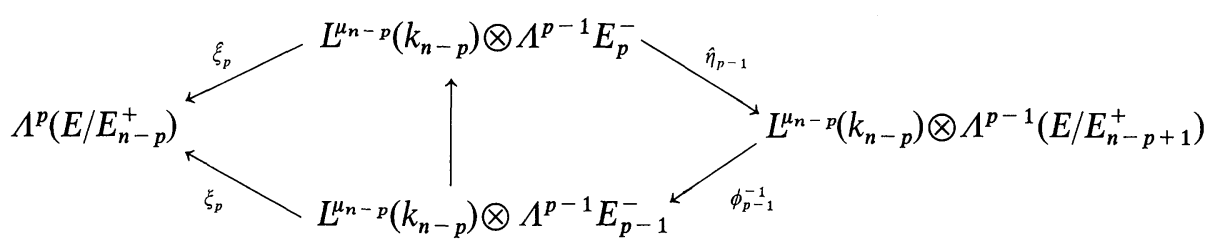

Proof. Now $L^{\mu_{n-p+1}}\left(k_{n-p}\right)=E_{p+1}^{-} / E_{p}^{-}$, so if $x \in E_{p+1}^{-}$and $y_{1}, \ldots, y_{p-1} \in E_{p}^{-}$then

$$
\xi_{p}\left(\left(x+E_{p}^{-}\right) \otimes \bigwedge_{j=1}^{p-1} y_{j}\right)=\left(x+E_{n-p}^{+}\right) \wedge \bigwedge_{j=1}^{p-1}\left(y_{j}+E_{n-p}^{+}\right) .
$$

As we are not on $S_{n-p+1}$ we have $E=E_{p-1}^{-} \oplus E_{n-p+1}^{+}$, and therefore

$$
E_{p}^{-}=E_{p-1}^{-} \oplus\left(E_{n-p+1}^{+} \cap E_{p}^{-}\right),
$$

so we let $y_{j}=y_{j}^{\prime} \oplus y_{j}^{\prime \prime}$ with respect to this decomposition, and then

$$
\left(\xi_{p} \circ \phi_{p-1}^{-1} \circ \hat{\eta}_{p-1}\right)\left(x+E_{p}^{-} \otimes \bigwedge_{j=1}^{p-1} y_{j}\right)=\left(x+E_{n-p}^{+}\right) \wedge \bigwedge_{j=1}^{p-1}\left(y_{j}^{\prime}+E_{n-p}^{+}\right) .
$$

Comparing (72) and (73) it suffices to show that $y_{j}^{\prime \prime} \in E_{n-p}^{+}$, then the diagram commutes. But $y_{j}^{\prime \prime} \in E_{n-p+1}^{+} \cap E_{p}^{-}$from (72) and this is a 1-dimensional subspace. As 
we are on $S_{n-p}$

so

$$
0 \neq E_{n-p}^{+} \cap E_{p}^{-} \subset E_{n-p+1}^{+} \cap E_{p}^{-}
$$

$$
y_{j}^{\prime \prime} \in E_{n-p+1}^{+} \cap E_{p}^{-}=E_{n-p}^{+} \cap E_{p}^{-}
$$

and we have $y_{j}^{\prime \prime} \in E_{n-p}^{+}$as required.

From this lemma $\hat{\xi}_{p}$ is determined by $\xi_{p}$ on $S_{n-p}-S_{n-p+1}$ but for a general monopole $S_{n-p} \cap S_{n-p+1}$ is finite and so by continuity $\xi_{p}$ is determined completely.

These results enable us to recover the bundle iteratively and we have proved Theorem (58) for $\mathrm{SU}(n)$.

To prove Theorem (58) for $\mathrm{Sp}(n)$ it is enough to embed $\mathrm{Sp}(n) \rightarrow \mathrm{SU}(2 n)$ in the standard way. The spectral curves $S_{1}, \ldots, S_{n}$ for $\mathrm{Sp}(n)$ give rise to the $2 n-1$ spectral curves $S_{1}, \ldots, S_{n-1}, S_{n}, S_{n-1}, \ldots, S_{1}$ for the $\mathrm{SU}(2 n)$ monopole. If we denote these curves by $T_{1}, \ldots, T_{2 n-1}$ respectively we see that a general $\mathrm{Sp}(n)$ monopole regarded as an $\mathrm{SU}(2 n)$ monopole has $T_{i} \cap T_{i+1}$ finite and therefore can be reconstructed by the methods used above.

For the $\mathrm{SO}(2 n)$ case we obtain a more complicated flag-inside the bundle $Q\left(\mathbb{C}^{2 n}\right)=E$. It has the form

$$
0 \subset E_{1}^{-} \ldots \subset E_{n-1}^{-} C_{E_{\bar{n}}}^{E \bar{n}_{n}} \subset E_{n+1}^{-} \subset \ldots \subset E_{2 n}^{-}=E .
$$

Using the same methods as for SU(n) we can reconstruct the flag up to $E_{-n}^{-}, E_{n}^{-}$and then $E_{n+1}^{-}$is determined by

$$
E_{n+1}^{-}=\frac{E_{n}^{-} \oplus E_{-n}^{-}}{E_{n-1}^{-}}
$$

and the rest of the reconstruction is straightforward.

The $\mathrm{SO}(2 n+1)$ monopole is not possible to do by these methods. The problem is that considered as an $\mathrm{SU}(2 n+1)$ monopole its spectral curves $S_{1}, \ldots, S_{n}$ become the $2 n$ curves $S_{1}, \ldots, S_{n}, S_{n}, \ldots, S_{1}$ and it is not a general $\mathrm{SU}(2 n+1)$ monopole. Other choices of representation lead to similar problems.

For the exceptional groups this same problem occurs so at present Theorem (58) is proved for the $\mathrm{SU}(n), \mathrm{Sp}(n)$, and $\mathrm{SO}(2 n)$ monopoles and I conjecture that it is true for the others.

\section{Non-Maximal Symmetry Breaking}

For the case of general symmetry breaking we have $\Phi^{\infty}: S^{2} \rightarrow K / C\left(T_{0}\right)$. If we fix a point $N \in S^{2}$ we can choose a maximal torus so that $\Phi^{\infty}(N),{ }^{*} F^{\infty}(N) \in L T$. Now in this case $\Phi^{\infty}=\Phi^{\infty}(N)$ doesn't determine a base of simple roots. However, we can consider the set of bases $\Delta$ that have the property that $\alpha_{i}\left(\Phi^{\infty}\right) \geqq 0 \forall \alpha_{i} \in \Delta$. This corresponds to choosing those fundamental Weyl chambers that have $\Phi^{\infty}$ in their closure. If we choose one of these, say $\Delta$, then the others are all related to it by the action of a subgroup $W_{0}$ of the Weyl group $W_{0}$. This is the subgroup of the Weyl group fixing $\Phi^{\infty}$ or $C\left(T_{0}\right) \cap N(T) / T$ the Weyl group of $C\left(T_{0}\right)$. 
Now if we order the simple roots in $\Delta$ so that we have

$$
\alpha_{i}\left(\Phi^{\infty}\right)>0, \quad 1 \leqq i \leqq s \quad \text { and } \quad \alpha_{i}\left(\Phi^{\infty}\right)=0, \quad s+1 \leqq i \leqq r,
$$

we can consider the set of fundamental weights $\lambda_{1}, \ldots, \lambda_{s}$. These are invariant under the action of $W_{0}$, in fact because $W_{0}$ is generated by the reflections $\sigma_{i}$ for $s+1 \leqq i \leqq r$, it suffices to consider $\sigma_{i}\left(\lambda_{j}\right)$ for $i>s$ and $j \leqq s$ but this is just $\lambda_{j}$. The topological charges of the monopole are therefore defined to be [6]

$$
2 \lambda_{i}\left({ }^{*} F^{\infty}\right), \quad 1 \leqq i \leqq s .
$$

The remaining fundamental weights are not invariant under $W_{0}$ so the magnetic charges are defined to be [6]

$$
\left\{\left(\omega \lambda_{i}\right)\left({ }^{*} F^{\infty}\right) \mid s<i \leqq r, \omega \in W_{0}\right\} .
$$

For this case the flag manifold $K / C\left(T_{0}\right)$ is diffeomorphic to the space $G / P$ where $P$ is a parabolic subgroup of $G$. The Lie algebra of $P$ is the subalgebra of $L G$ generated by $L B$ and all the $L_{\alpha_{i}}$ for $s+1 \leqq i \leqq r$.

The twistor correspondence of Sect. 2 generalizes in the obvious way to give a holomorphic bundle $Q$ with two reductions $R^{+}, R^{-}$to the parabolic subgroups $P$ and $\bar{P}$ respectively.

As an example we consider minimal symmetry breaking for $\mathrm{SU}(n)$. We consider a Higgs field of the form

$$
\Phi=i \operatorname{diag}\left(\mu_{1}, \mu_{2}, \ldots, \mu_{2}\right)-\frac{i}{2 r} \operatorname{diag}\left(k_{1}, \ldots, k_{n}\right)+O\left(\frac{1}{r^{2}}\right),
$$

where $\mu_{1}>\mu_{2}$ and $(n-1) \mu_{2}+\mu_{1}=0=\sum k_{i}$. The Weyl group $W=S_{n}$, the group of permutations of $\{1,2, \ldots, n\}$ and $W_{0}$ is the subgroup leaving 1 fixed. A base for which $\alpha_{i}\left(\Phi^{\infty}\right) \geqq 0$ is

$$
\Delta=\left\{\left(x_{1}-x_{2}\right),\left(x_{2}-x_{3}\right), \ldots,\left(x_{n-1}-x_{n}\right)\right\}=\{(1,-1,0, \ldots), \ldots,(0,0, \ldots, 1,-1)\},
$$

and the associated fundamental weights are

$$
\{(1,0, \ldots),(1,1,0, \ldots),(1,1,1, \ldots), \ldots,(1,1, \ldots, 0)\} .
$$

The subgroup $W_{0}$ clearly only fixes $(1,0, \ldots)$ so the topological charge is $k_{1}$.

The parabolic subgroup $P$ is the subgroup of $\operatorname{SL}(n, \mathbb{C})$ fixing a line in $\mathbb{C}^{n}$ and $\bar{P}$ is the subgroup fixing the orthogonal plane. So if we form the associated vector bundle $E=Q\left(\mathbb{C}^{n}\right)$ it has a line sub-bundle $E_{1}^{+}$and a hyperplane sub-bundle $E_{n-1}^{-}$. By applying the methods used to prove Theorem (21), these are seen to satisfy

$$
\begin{gathered}
E_{1}^{+} \simeq L^{\mu_{1}}\left(-k_{1}\right), \\
E / E_{1}^{+} \simeq L^{\mu_{2}}\left(-k_{2}, \ldots,-k_{n}\right), \\
E_{n-1}^{-} \simeq L^{\mu_{2}}\left(k_{2}, \ldots, k_{n}\right), \\
E / E_{n-1}^{-} \simeq L^{\mu}\left(k_{1}\right), \quad \text { where } O\left(p_{1}, \ldots, p_{m}\right)=O\left(p_{1}\right) \oplus \ldots \oplus O\left(p_{m}\right) .
\end{gathered}
$$

The spectral curve $S$ of this monopole is defined by

$$
\phi: E_{1}^{+} \rightarrow E / E_{n-1}^{-},
$$


and $S$ is in the linear system $\left|O\left(2 k_{1}\right)\right|$. The fundamental diagram is then

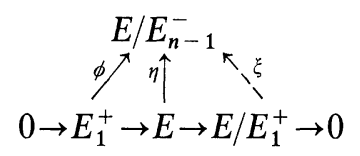

or

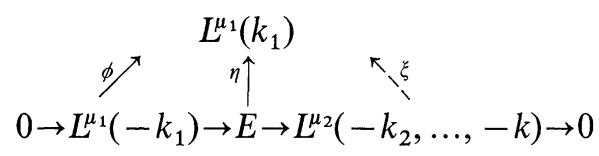

and it follows that $\xi$ is a section over $S$ of $L^{\mu_{1}-\mu_{2}}\left(k_{1}+k_{2}, \ldots, k_{1}+k_{n}\right)$.

For this monopole the spectral data is defined to be $S$ and the section $\xi$. It is immediate from Lemma (57) that $E$ can be recovered from the spectral data. From the comments on endomorphisms at the beginning of Sect. 4 this means that the monopole is determined by the spectral data.

The other minimal SU(n) case is to take $\lambda_{1}<\lambda_{2}$ and this is treated similarly. Putting these together we have proved

Theorem (81). The irreducible minimal $\mathrm{SU}(n)$ monopole is determined by its spectral data.

To see what is happening from the flag-manifold point of view we consider the action of $\bar{P}$ on $G / P$. Now $G / P$ is just $\mathbb{C P}_{n-1}$, complex projective $n-1$ space and the action of $\bar{P}$ divides it into two orbits, the lines intersecting the plane fixed by $\bar{P}$ and the lines not intersecting this plane. The first set is a copy of $\mathbb{C P}_{n-2}$ and the other its open complement.

Now we can form the bundle $Q\left(\mathbb{C P}_{n-1}\right)=R^{+}\left(\mathbb{C P}_{n-1}\right)=R^{-}\left(\mathbb{C P}_{n-1}\right)$. As $\bar{P}$ acting on $\mathbb{C P}_{n-1}$ preserves the decomposition above $R^{-}\left(\mathbb{C P}_{n-1}\right)$ has a decomposition in each fibre. On the other hand $P$ preserves a point in $\mathbb{C P}_{n-1}$ so $R^{+}\left(\mathbb{C P}_{n-1}\right)$ has a section. If we intersect this section with the decomposition above and project onto $\mathscr{T}$ we obtain the curve $S$.

In the general case because $L P$ contains $L_{-\alpha_{j}}$ for $s<j \leqq r$ the highest weight space $V_{\lambda_{i}}\left(\lambda_{i}\right) \subset V_{\lambda_{i}}$ is stable under $P$ only when $1 \leqq i \leqq s$. This means we can define $s$ spectral curves as in Sect. 3. It is not clear what the remainder of the spectral data should be. I would expect a combination of the two cases we have looked at, that is, some sections of higher rank bundles and some divisors on the curves, as these are the two "extreme" cases.

\section{Summary}

We have seen that the spectral data determines the $\mathrm{SU}(n), \mathrm{Sp}(n)$, and $\mathrm{SO}(2 n)$ monopole for the maximal symmetry breaking case and an $\mathrm{SU}(n)$ monopole for the minimal symmetry breaking case. It would be nice to have a method of proof that worked for all groups and all symmetry breaking. This would seem to require either working with the adjoint representation or reconstructing the principal bundle directly. 
In a recent paper [13] Nahm has shown how to define $(n-1)$ algebraic curves for an $\mathrm{SU}(n)$ monopole. As yet I am unable to show that this curves are the spectral curves although it seems highly plausible.

Acknowledgements. The author would like to thank Professor Sir Michael Atiyah for his help and inspiration and Dr. N. Hitchin for many useful discussions, in particular the idea behind Proposition 51. The financial support of the Association of Commonwealth Universities and the hospitality of the Mathematical Sciences Research Institute are also gratefully acknowledged.

\section{References}

1. Atiyah, M.F., Bott, R.: The Yang-Mills equations over Riemann surfaces. Philos. Trans. R. Soc. (London) A 308, 523-615 (1982)

2. Atiyah, M.F., Hitchin, N.J., Singer, I.M.: Self-duality in four-dimensional Riemannian geometry. Proc. R. Soc. (London) A 362, 425-561 (1978)

3. Bernstein, I.N., Gel'fand, I.M., Gel'fand, S.I.: Schubert cells and cohomology of the spaces $G / P$. Russ. Math. Surv. 28:3, 1-26 (1973)

4. Bogolmolny, E.B.: The stability of classical solutions. Sov. J. Nucl. Phys. 24:4, 449-454

5. Coppel, W.A.: Stability and asymptotic behaviour of differential equations. Boston, DC: Heath and Company 1965

6. Goddard, P., Nuyts, J., Olive, D.I.: Gauge theories and magnetic charge. Nucl. Phys. B 125, 128 (1977)

7. Eastwood, M.G., Penrose, R., Wells, R.O.: Cohomology and massless fields. Commun. Math. Phys. 78, 305-351 (1981)

8. Griffiths, P., Harris, J.: Principles of algebraic geometry. New York: Wiley 1978

9. Hitchin, N.J.: Monopoles and geodesics. Commun. Math. Phys. 83, 579-602 (1982)

10. Hitchin, N.J.: On the construction of monopoles. Commun. Math. Phys. 89, 145-190 (1983)

11. Koboyashi, S., Nomizu, K.: Foundations of differential geometry. Vols. 1 and 2. New York: Interscience 1969

12. Murray, M.K.: Monopoles and spectral curves. Commun. Math. Phys. 90, 263-271 (1983)

13. Nahm, W.: The algebraic geometry of multimonopoles. Bonn, Preprint, HE-82-30

14. Taubes, C.H.: The existence of multi-monopole solutions to the non-abelian, Yang-MillsHiggs equations for arbitrary simple gauge groups. Commun. Math. Phys. 80, 343-367 (1981)

15. Warner, G.: Harmonic analysis on semi-simple Lie groups 1. Berlin, Heidelberg, New York: Springer 1972

16. Weinberg, E.: Fundamental monopoles and multimonopole solutions for arbitrary simple gauge groups. Nucl. Phys. B 167, 500 (1980)

Communicated by A. Jaffe

Received June 4, 1984; in revised form August 6, 1984 